\title{
Determination of dsRNA interactome upon Sindbis virus infection in human cells identifies SFPQ as a critical proviral factor
}

Erika Girardi ${ }^{1, *}$, Mélanie Messmer ${ }^{1}$, Paula Lopez ${ }^{1}$, Aurélie Fender ${ }^{1}$, Johana Chicher ${ }^{2}$, Béatrice Chane-Woon-Ming ${ }^{1}$, Philippe Hammann ${ }^{2}$, Sébastien Pfeffer ${ }^{1, *}$

${ }^{1}$ Université de Strasbourg, Architecture et Réactivité de l'ARN, Institut de Biologie Moléculaire et Cellulaire du CNRS, 2 allée Konrad Roentgen, 67084 Strasbourg France

${ }^{2}$ Université de Strasbourg, Institut de Biologie Moléculaire et Cellulaire du CNRS, Plateforme Protéomique Strasbourg - Esplanade, 2 allée Konrad Roentgen, 67084 Strasbourg France

* To whom correspondence should be addressed: e.girardi@ibmc-cnrs.unistra.fr; s.pfeffer@ibmc-cnrs.unistra.fr

Keywords: dsRNA, proteomics, Sindbis virus, dsRNAome, SFPQ, RNA binding protein

Running title: dsRNA interactome of Sindbis virus infected cells 


\begin{abstract}
Viruses are obligate intracellular parasites, which depend on the host cellular machineries to replicate their genome and complete their infectious cycle. Long double stranded (ds)RNA is a common viral by-product originating during RNA virus replication and is universally sensed as a danger signal to trigger the antiviral response. As a result, viruses hide dsRNA intermediates into viral replication factories and have evolved strategies to hijack cellular proteins for their benefit. The characterization of the host factors associated to viral dsRNA and involved in viral replication remains a major challenge to develop new antiviral drugs against RNA viruses. Here, we performed anti-dsRNA immunoprecipitation followed by mass spectrometry to fully characterize the dsRNA interactome in Sindbis virus (SINV) infected human HCT116 cells. Among the validated factors, we characterized SFPQ (Splicing factor, proline-glutamine rich) as a new dsRNA-associated factor upon SINV infection. We proved that SFPQ is able to directly bind to dsRNAs in vitro, that its association to dsRNA is independent of single-stranded (ss)RNA flanking regions in vivo and that it is able to bind to the viral genome upon infection. Furthermore, we showed that both knock-down and knockout of SFPQ reduce SINV infection in human HCT116 and SK-N-BE(2) cells, suggesting that SFPQ could enhance viral replication. Overall, this study not only represents a resource to further study SINV dsRNA-associated factors upon infection but also identifies SFPQ as a new proviral dsRNA binding protein.
\end{abstract}




\section{Introduction}

Because of their strict dependency on the host cell machinery to replicate, viruses interact with many host factors to ensure the efficient and successful completion of their life cycle. The least complex the viral genome is, the more the virus will rely on cellular proteins. For instance, positive single stranded (ss)RNA viral genomes, which mimic cellular mRNA features, typically encode for a handful of proteins able to replicate and package their genome. As a consequence, these viruses are known to hijack host factors, such as regulatory non-coding RNAs (1) as well as several cellular RNA Binding Proteins (RBPs) $(2,3)$ causing profound alterations in the cell.

Canonical RBPs participate in every step of virus infection, from the recognition of the viral RNA to its replication and translation (2). In particular, a broad range of RNA viruses are able to hijack nuclear shuttling host RBPs and to alter their roles to support their own replication, thereby indirectly impacting host gene expression (4). Among the most complex interactions between viral RNA and cellular proteins are those needed for the formation of intracellular structures known as 'viral factories', which are not only necessary to allow viral replication, but also to protect the viral genome from detection and clearance by the antiviral response (5). Indeed, in order to counteract the infection, the cell evolved an arsenal of antiviral factors able to recognize foreign viral features. Thus, the absence of a 5' cap, or the presence of double stranded (ds)RNA structures longer than 30 base pairs (bps), will trigger a potent and evolutionary conserved innate immune response. Long viral dsRNA in particular constitutes a universal danger signal in higher eukaryotes, as it accumulates invariably and specifically as a by-product of viral replication or transcription. Antiviral RNA interference (RNAi) constitutes the major antiviral response in plants and invertebrates. It is elicited by recognition and cleavage of long viral dsRNA molecules into small interfering RNAs (siRNA) by the ribonuclease Dicer. SiRNAs are then loaded into effector complexes comprising a member of the Argonaute (Ago) family, where they act as guides to degrade viral RNAs via sequence complementarity and restrict the infection (6). The cellular response to viruses can also be triggered by foreign nucleic acids in vertebrates, but it rather culminates with the induction of type I Interferons (IFNs) and of hundreds of interferon-stimulated-genes (ISGs). Such a chain of events perturbs the viral replication but also promotes the inhibition of protein synthesis, cellular RNA degradation and, ultimately, cell death (7). Several dsRNA sensors, such as RIGI, MDA5 or LGP2, and other dsRNA Binding Proteins (dsRBPs) such as PKR, ADAR and OAS proteins have been well characterized so far and their general role during viral infection 
has been identified (8). Nonetheless, additional cellular factors, such as RNA helicases, are also emerging as general or virus-specific players in the recognition and immune defence to viral dsRNA (9). Therefore, the identification of such host factors is crucial in order to better understand dsRNA sensing in viral infections and to study the interactions between the host and its viruses.

Sindbis virus (SINV) is a member of the alphavirus genus from the Togaviridae family. It has a capped and polyadenylated positive-sense RNA genome of $12 \mathrm{~kb}$, which consists of two open reading frames (ORFs). ORF1 encodes the non-structural polyprotein and ORF2 encodes the structural polyprotein. While the structural proteins are translated from a sub-genomic RNA, the non-structural proteins (nsP1, nsP2, nsP3 and nsP4) are directly translated from the genomic RNA since they are necessary for replication of the viral RNA inside the cytoplasm of host cells. This occurs through the synthesis of a complementary antigenomic RNA and the accumulation of long dsRNA intermediates in the cytoplasm of infected cells (10).

Alphavirus infection strongly affects many cellular pathways to favour viral production and interfere with the antiviral response. For instance, arthritogenic alphavirus nsP3 protein has been shown to interact with and modulate the activity of proteins related to stress granule formation, such as G3BP1. Proteomic studies performed to characterize the protein complexes associated with the SINV viral protein nsP3 (11) or the RNA-dependent RNA polymerase nsP4 (12) assessed that the interaction with G3BP1 and G3BP2 is important for the formation and function of SINV RNA genome replication complexes. Old world alphaviruses' nsP2 protein has been shown to inhibit RNA polymerase II transcription (13) and to contribute to the shutoff of CAP-dependent translation (14). Moreover, the viral RNA itself is able to interfere with host mRNA translation, being able to act as a sponge and sequester the nuclear shuttling protein HuR/ELAV1 (15), as well as to hijack cellular microRNAs $(16,17)$.

System-wide approaches to discover the cellular RBP interactome of viral RNA are emerging as key to deepen our understanding of the involvement of host proteins in infected cells (18). In particular, many efforts have been made in order to identify host factors able to bind to the alphavirus viral RNA $(19,20)$ and to elucidate the role of these factors at different stages of the viral life cycle. Recently, RNA interactome capture (RNA-IC) has also been employed to identify the cellular RNA-binding proteome associated with SINV viral RNA and found out several RBPs which may regulate viral replication and could be targeted to modulate the infection (21). However, these approaches are focused on genomic or messenger viral RNAs and are not able to reliably identify proteins interacting with replication intermediates, which 
are one of the key features triggering the cell immune response. Here, we aimed at identifying specifically the long dsRNA interactome upon SINV infection in human cells in an unbiased manner and at characterizing the relevance of dsRNA-associated proteins during infection. To this end, we employed an approach based on dsRNA immunoprecipitation using a dsRNAspecific antibody followed by proteomic analysis and identified SFPQ as a new proviral, dsRNA-associated factor upon SINV infection. 


\section{Results}

\section{Identification of the dsRNA interactome in mock- and SINV-infected human cells.}

SINV genomic RNA replicates through the production of a reverse complementary antigenomic RNA, which in turn causes the accumulation of long dsRNA intermediates in the cytoplasm of infected cells (Figure 1A). The presence of long dsRNAs was verified in human colon carcinoma HCT116 cells infected with SINV for $24 \mathrm{~h}$ at an MOI of 0.1 compared to uninfected ones by immunofluorescence assay using the anti-dsRNA (J2) antibody, which specifically recognizes dsRNAs longer than $40 \mathrm{bp}$ (22)(23) (Figure 1B).

In order to characterize the long dsRNA interactome in mock and SINV infected HCT116 cells, we adapted the method of immunoprecipitation of long RNA duplexes (24) to the analysis of dsRNA-associated proteins. The experimental design was based on the pull-down of long dsRNAs from cellular lysates of mock-infected or SINV-GFP-infected HCT116 cells for $24 \mathrm{~h}$

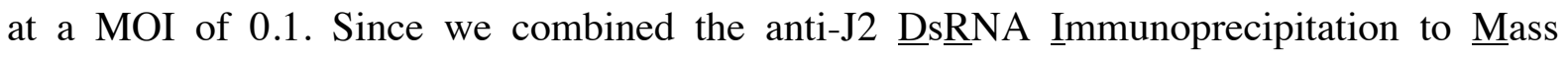
Spectrometry of the isolated dsRNA-associated protein complexes, we named this approach DRIMS (Figure 1C). The presence of viral RNA specifically immunoprecipitated in the infected samples was double-checked by RT-qPCR on RNA extracted from the J2- and control IgG -IP (Figure 1D).

Protein profiles in total extracts, J2 and IgG eluates were analysed by silver staining of polyacrylamide gels (Figure S1A) and J2- and IgG-IP samples were then analysed by liquid chromatography coupled to tandem mass spectrometry (LC-MS/MS). After identification of viral and human proteins in each sample, hierarchical clustering analysis of the J2-IP replicates identified two distinct groups, indicating higher similarities in the protein profiles among the mock and SINV samples, respectively (Figure S1B). Differential expression analysis was then performed by comparing J2-IP samples in SINV-infected versus mock-infected cells and revealed the enrichment of specific host and viral proteins upon viral infection (Figure 1E and Table S1). Protein enrichment of the J2-IP over the IgG-IP samples upon SINV-GFP infection showed enrichment profiles similar to SINV J2-IP versus mock J2-IP ones (Figure S1C), while comparison of the J2-IP to IgG-IP samples in mock conditions revealed very few significantly enriched proteins, potentially associated to endogenous dsRNAs (Figure S1D).

We identified a total of 63 significantly enriched proteins in SINV-GFP infected J2-IP samples compared to mock, validated with $\mathrm{FDR}<1 \%$, having at least 5 spectral counts in the most abundant condition, a fold change of at least 2 and an adjusted p-value lower than 0.05 (Table S1). As expected, viral proteins were specifically enriched in the SINV J2-IP compared to the 
mock J2-IP (Figure 1E), as well as to the IgG-IP controls (Figure S1C). Among the most enriched cellular proteins in infected cells, we validated the presence of known dsRNAassociated factors involved in antiviral innate immunity to long dsRNAs, such as ADAR1 (DSRAD), DHX9, PKR (E2AK2) and PACT (PRKRA). Interestingly, we also identified DICER1 as specifically associated to dsRNAs upon SINV infection. Since the J2-Ab was previously shown to be unable to recognize pre-miRNA structures (25), this indicates a potential role of DICER1 in the recognition of long dsRNAs in infected human cells.

In addition, several nuclear RBPs known to be involved in pre-mRNA metabolism, such as NONO/p54nrb, SFPQ/PSF, RBM14, PSPC1 and MATR3, showed a 1.5 to $5 \log 2$ fold change enrichment in the SINV J2-IP compared to either the SINV IgG-IP or the mock J2-IP (Figure $1 \mathrm{E}$ and S1C). In particular, NONO and SFPQ, which belong to the conserved family of DBHS (Drosophila behaviour human splicing) proteins and can form a heterodimer, were already enriched in mock J2-IP versus IgG-IP samples (Figure S1D) and their association with long dsRNAs seemed to increase upon infection (Figure 1E).

Gene Ontology (GO) term enrichment analysis of the proteins overrepresented in SINV J2-IP samples based on molecular function, cellular components and biological processes indicated that most of these proteins are RNA binding proteins (RBPs) (Figure 2A, left panel), present in ribonucleoprotein granules (Figure 2A, middle panel) and are involved in pre-mRNA processing/metabolism (Figure 2A, right panel). Moreover, STRING analysis showed that these enriched cellular proteins mostly belong to known interaction networks either in the cytoplasm (red) or in the nucleus (blue) (Figure 2B).

In order to validate the dsRNA association of the enriched proteins identified by DRIMS, we performed western blot analysis on J2-IP compared to IgG-IP samples in mock and SINVinfected HCT116 cells and confirmed an exclusive or increased interaction of PACT, PKR, ADAR1, DHX9, DICER, MATR3 and SFPQ with dsRNA upon SINV infection (Figure 2C). Overall, our data suggest that the DRIMS approach is a powerful way to identify long dsRNA interactome upon viral infection in human cells and that beside known antiviral factors it allowed us to isolate several novel RBPs potentially involved in dsRNA recognition during SINV infection.

\section{SFPQ binds both to synthetic dsRNA in vitro and to viral RNA in vivo}

Paraspeckles are dynamic ribonucleoprotein (RNP) nuclear bodies built by protein liquidliquid phase transition and defined by the presence of the structural lncRNA NEAT1 (nuclear- 
enriched abundant transcript 1) and the NEAT1-binding paraspeckle proteins SFPQ, NONO and PSPC1. Several other proteins and RNAs can be sequestered in these granules resulting either in the inhibition or enhancement of their molecular function (26). For instance, hyperedited RNAs containing Alu inverted repeats (IRAlus) can be specifically retained within nuclear paraspeckles in order to impair their export and translation. Paraspeckle changes in size, composition and number have already been described under stress condition and, more recently, in response to certain viral infections (27). However, a direct link between paraspeckle proteins and their binding to viral dsRNA has not been investigated so far.

Among the dsRNA-enriched proteins retrieved in SINV-infected cells, we found several factors which can be part of paraspeckles and previously described as associated to pre-RNA metabolism (Figure 1E, light blue). In order to confirm their localization and verify whether any change in paraspeckles was induced upon SINV infection in HCT116 cells, we performed immunofluorescence analysis against three of these proteins MATR3, NONO and SFPQ. As a control, we also tested the localization of G3BP1 and IGFBP2, which are known to be present in the cytoplasm in virus-induced stress granules and were found as enriched in SINV-J2-IP by DRIMS. Moreover, we used antibodies against the non-structural viral protein nsP2 and the cellular RBP ELAV1, which is known to be hijacked by the SINV viral RNA to the cytoplasm, to validate the infection in HCT116 cells (Figure S2). Our results also validate the nuclear localization of paraspeckle-associated proteins and the increased signal intensity upon infection especially for NONO, which is part of the paraspeckle core. However, we noticed some cytoplasmic re-localization of SFPQ in SINV-infected cells.

Beside its localization within paraspeckles as a heterodimer with NONO, SFPQ is a multifunctional protein involved in several vital cellular processes, such as transcription or premRNA processing and localization. Thus, it is known to bind to single stranded RNA and more particularly to the poly-pyrimidine tract upstream of intronic 3'splice site (ss) $(28,29)$ to assist spliceosome assembly, but also to C-rich sequences close to the $5^{\prime}$ splice sites of long introns therefore regulating long intron-containing gene expression (30). Since we retrieved SFPQ enriched on dsRNA upon infection by DRIMS (Figure 1E, 2C, Table S1), we first asked whether such a binding could take place in vitro and independently of its interaction with NONO which is known to directly bind to edited host dsRNA (31). To this end, we performed electrophoretic mobility shift assay (EMSA) using a commercial recombinant SFPQ protein, which was verified by silver staining analysis (Figure S3A). After incubation of increasing amount of SFPQ with a 65 bp-long, in vitro transcribed and radiolabelled dsRNA, we could 
visualize a specific shift, which was lost by addition of the corresponding cold dsRNA competitor (dsRNA-EGFP) but not after addition of a non-specific yeast-tRNA (Figure 3A). This result supports the hypothesis that SFPQ can bind to long dsRNAs in vitro.

To further characterize the SFPQ-dsRNA interaction, we immunoprecipitated the endogenous SFPQ in mock and SINV infected HCT116 cells and isolated the associated RNAs (RNAimmunoprecipitation, RIP). After verifying the immunoprecipitation of SFPQ by western blot analysis (Figure S3B), we performed a dot blot analysis using the J2-antibody to visualize long dsRNA molecules (Figure 3B). While uninfected cells did not show any dsRNA accumulation, we observed a specific signal both in total RNA (INPUT) and J2-IP upon SINV infection compared to the IgG-IP, suggesting that the association of dsRNA species with SFPQ takes place specifically in infected cells. Additionally, we showed by RT-qPCR that the viral genomic RNA is specifically enriched in the SFPQ-RIP compared to the IgG-IP (Figure 3C). These data confirm the enrichment of SFPQ on dsRNAs upon SINV infection observed by DRIMS (Figure 1E) and validated by western blot (Figure 2C).

In order to exclude that SFPQ association to dsRNA is mediated by its binding to flanking single stranded (ss)RNA regions, we performed J2-IP experiments followed by on-beads RNase T1 treatment before elution (Figure 3D and S3C). We could confirm the efficiency of RNAse T1 digestion on total RNA (INPUT) (Figure S3C) and that the association of PKR (dsRBP, positive control) was unchanged before and after treatment, while Ago2 was not enriched (ssRBP, negative control). Interestingly, enrichment of SFPQ in the J2-IP upon infection remained unchanged before and after RNase T1 digestion, suggesting a direct binding to dsRNA regions in infected cells (Figure 3D). Finally, we performed SFPQ coimmunofluorescence analysis in mock and SINV infected HCT116 cells. Despite the prevalent nuclear localization and the absence of a measurable colocalization, SFPQ was also present in the cytoplasm of infected cells where the viral dsRNA accumulates (Figure 3E).

Overall, these findings not only indicate that SFPQ is able to bind dsRNA in vitro and is associated to dsRNA in vivo upon infection, but also that this seems to happen independently of its interaction with NONO within paraspeckles and may take place in the cytoplasm where the viral RNA replicates.

\section{SFPQ depletion strongly reduces SINV infection in HCT116 and SK-N-BE(2) cells.}

To further decipher the involvement of SFPQ and the other identified paraspeckle-associated factors in the response to dsRNA upon SINV infection, we performed an siRNA-based loss- 
of-function analysis and quantified the effect of specific gene depletion on viral infection by SINV-GFP. Using this strategy, we could demonstrate that knock-down (KD) of SFPQ significantly reduces GFP expression in HCT116 infected cells (Figure 4A). This effect was similar to the one observed upon transfection of virus-specific siRNAs (siSINV) and showed an opposite phenotype to the one observed using siRNA targeting PKR (Figure 4A). In agreement with the decrease in GFP, the levels of viral CAPSID (Figure 4B), viral titers (Figure 4C) and viral genomic RNA (Figure 4D) were reduced upon SFPQ KD compared to the control (siCTRL).

We validated these findings in another cell type suitable for SINV infection, namely the SK$\mathrm{N}-\mathrm{BE}(2)$ human neuroblastoma cells, which can be differentiated into neurons upon retinoic acid (RA) treatment (Figure S4). We observed not only the decrease in GFP by epifluorescence microscopy (Figure S4A), but also a reduction in the levels of viral CAPSID (Figure S4B) and viral titers (Figure S4C) upon SFPQ KD compared to the control (siCTRL).

Finally, we generated a heterozygous (+/-) SFPQ knock-out (KO) HCT116 cell line by CRISPR/Cas9 (Figure 5A-B) and we tested the effect of SFPQ depletion independently of siRNAs. Although one allele remained wild-type, due to the fact that the gene may be essential, we could validate the negative impact of SFPQ reduction on GFP expression levels, CAPSID levels and viral titers at 24 hpi compared to WT cells (Figure 5C-E).

Overall, our results demonstrate a functional effect of SFPQ depletion on SINV infection in two independent cell types and with two different loss-of function methods, suggesting that SFPQ could play a pro-viral role during infection. 


\section{Discussion}

Unbiased proteome-wide approaches have proven to be very useful to find new host factors regulated by or able to regulate viral infections. Over 2000 cellular RBPs have been described so far (32), but understanding which RBP participates to the life cycle of a virus and at which step remains a challenge. Here, we showed that our DRIMS approach is very effective to identify dsRNA associated proteins, since it allowed us to validate the presence of proteins with canonical dsRNA binding domains (dsRBDs) or helicase domains (9) involved in antiviral innate immunity, such as PKR, PACT, Staufen, ILF2, ILF3 and DHX9 associated to long dsRNA upon SINV infection in human cells. Interestingly, we also identified factors such as DICER or ADAR1, suggesting that SINV viral RNA duplexes are a potential target for enzymes acting on dsRNA, although we did not test the functionality of these interactions. Whether DICER is indeed able to cleave long dsRNAs and generate siRNAs or simply to associate with the substrate remains to be clarified in this context.

Surprisingly, we were not able to find expected innate immune dsRNA sensors of the RIG-I like receptor (RLR) family, such as RIG-I or MDA5 using our approach. One hypothesis could be that their association to the substrate is too stable and does not allow the J2-antibody to recognize and isolate the long dsRNA. Alternatively, a relative low concentration of RIG-I and MDA5 (33) may explain why they would not represent preponderant actors in the recognition of SINV dsRNA compared to other factors such as PKR in the cellular context used here. We did however isolate all four viral nsPs in SINV-infected cells suggesting that the DRIMS approach is suitable for the isolation of proteins bound to the replication complex itself.

Increasing evidences show that host factors without typical protein domains known to bind to ss or dsRNA are still able to associate with foreign nucleic acids and act as RNA co-sensors by modulating canonical dsRNA sensor activity (34). For instance, several hnRNP family members can negatively regulate RLR signaling. Upon viral infection, HNRNPE2 translocates from the nucleus to the cytoplasm where it interacts with MAVS to mediate its degradation (35). HNRNPM, which shuttles as well from the nucleus to the cytoplasm in infected cells, impairs the RNA-binding activity of RLRs (36). Finally, HNRNPC protects cells from accumulating RIG-I-stimulatory endogenous Alu-rich dsRNA (37).

Although alphavirus replication proceeds in the cytoplasm of infected cells and does not directly depend on nuclei, this family of viruses has the ability to alter subcellular localization of host nuclear RBPs (4). The abundance of nuclear proteins isolated with our approach, many of which with a known localization within paraspeckles, suggested that some of these factors 
may also localize to the cytoplasm upon SINV infection to favour or inhibit the viral cycle and possibly modulate the innate immune response.

One of such proteins we identified was SFPQ, an ubiquitous and abundant protein implicated in many aspects of nucleic acid biology in the nucleus, ranging from genome stability to transcription, as well as pre-mRNA splicing and 3'end RNA processing or nuclear retention (27). Although SFPQ is strongly localized in the nucleus under normal circumstances, several reports have shown that it may also have important non-nuclear functions such as mRNA posttranscriptional regulation and RNA granule formation especially in neurons (38).

Accordingly, when we assessed SFPQ localization before and after SINV infection in HCT116 cells by immunofluorescence, we observed that its accumulation occurs both in the nucleus and in the cytoplasm, which was compatible with its association with viral RNA upon infection. Moreover, we demonstrated that SFPQ expression has a significant impact on SINV infection, since SFPQ knock-down causes a reduction in SINV viral RNA and protein levels as well as in viral production. This phenotype was reproducible in two independent cell types (HCT116 and SK-N-BE(2) cells) and in SFPQ +/- knockout cells, suggesting a proviral role for SFPQ during SINV infection. It is worth to mention that $S F P Q$ seems to be an essential gene (27), which could explain why we were not able to generate a homozygous knock-out cell line.

A previous link between SFPQ and viral infection has already been reported for several viruses. For instance SFPQ interacts with HIV viral RNA and favours its nuclear-cytoplasmic export (39), and it also increases the efficiency of influenza virus mRNA polyadenylation (40). Moreover, SFPQ proviral effect has been reported during Hepatitis delta virus (HDV) (41), human rhinovirus A16 (HRV16) (42) and encephalomyocarditis virus (EMCV) infections (43). A recent study revealed as well a direct role of SFPQ on antiviral innate immunity showing that paraspeckle proteins may couple both transcription and post-transcriptional events. Indeed, SFPQ acts as a transcriptional repressor on the promoter of certain immune related genes and its re-localization to the core of paraspeckles upon infection leads to the induction of the antiviral gene program (44).

Among the proteins isolated by DRIMS, we also enriched for stress granules related factors. Stress granules are cytoplasmic RNP granules formed by mRNAs stalled in translation initiation and contain various translation initiation factors, a variety of RNA-binding proteins, and many non-RNA-binding proteins. During viral infection, stress granules recruit numerous antiviral proteins including RIG-I or PKR and enhance the induction of the innate immune 
response and viral resistance (45). Proteins from stress granules such as G3BP1 are also able to directly bind to viral dsRNA (46).

Due to their distinct localisation, nuclear and cytoplasmic RNP granules have long been considered independent entities. Recently, a crosstalk between stress granules and paraspeckles has been proposed in which stress granules would act as positive regulators of nuclear RNP granule assembly in response to stress. In particular, an overlap between the proteomes of paraspeckles and stress granules has been observed and it suggests that cytoplasmic granule formation may regulate paraspeckle assembly via sequestration or release of specific proteins. $(47,48)$. Although it is not clear if this interconnection exists in response to viral infection, it could explain the reason why we identified SFPQ as associated to dsRNA upon infection.

Moreover, we showed that SFPQ binds synthetic long dsRNA in vitro independently of its paraspeckle cofactor NONO. However, whether the dsRNA bound by SFPQ upon infection is specifically the viral replication intermediate or whether it is of cellular origin remains to be assessed. Indeed, we cannot exclude that host dsRNA accumulation specifically occurs in response to viral stress. As paraspeckles are involved in nuclear retention of edited transcripts containing dsRNA structures, it is tempting to imagine that binding of SFPQ to viral RNA in the cytoplasm could change paraspeckles composition and indirectly affect localization and expression of certain genes upon infection. 


\section{Material and Methods}

\section{Cell culture, viral stocks and virus infection}

Cell lines were maintained at $37^{\circ} \mathrm{C}$ in a humidified atmosphere containing $5 \% \mathrm{CO}_{2}$. HCT116, BHK-21 and Vero cells were cultured in Dulbecco's Modified Eagle medium (DMEM; Invitrogen) supplemented with 10\% FBS. SK-N-BE(2) cells (95011815, Sigma-Aldrich) were maintained in 1:1 medium composed of Ham's F12 medium (Gibco, Thermo Fisher Scientific Inc.) supplemented with 15\% FBS and Eagle's Minimal Essential Medium (ENEM) supplemented with 1\% NEAA (Gibco, Thermo Fisher Scientific Inc.). SK-N-BE(2) cell differentiation was induced by $10 \mu \mathrm{M}$ retinoic acid (RA) treatment (R2625, Sigma-Aldrich) for 5 days. Plasmids carrying a green fluorescent protein (GFP)-SINV genomic sequence or wild-type SINV genomic sequence (kindly provided by Dr Carla Saleh, Institut Pasteur, Paris, France) were linearized and used as a substrate for in vitro transcription using mMESSAGE mMACHINE capped RNA transcription kit (Ambion, Thermo Fisher Scientific Inc.) as in (17). Viral stocks were prepared in BHK-21 baby hamster kidney cells, and titers were measured by plaque assay in Vero cells. Cells were infected at a MOI of $10^{-1}$ and samples were harvested at 24 hours post-infection (hpi) unless specified otherwise.

\section{Standard plaque assay}

Vero cells seeded either in 96- or 24-well plates were infected with viral supernatants prepared in cascade 10-fold dilutions for $1 \mathrm{~h}$. Afterwards, the inoculum was removed and cells were cultured in $2.5 \%$ carboxymethyl cellulose for $72 \mathrm{~h}$ at $37^{\circ} \mathrm{C}$ in a humidified atmosphere of $5 \%$ $\mathrm{CO} 2$. Plaques were counted manually under the microscope. For plaque visualization, the medium was removed, cells were fixed with $4 \%$ formaldehyde for $20 \mathrm{~min}$ and stained with 1x crystal violet solution (2\% crystal violet (Sigma-Aldrich), $20 \%$ ethanol, $4 \%$ formaldehyde).

\section{DsRNA Immunoprecipitation and Mass Spectrometry (DRIMS)}

Protein G Dynabeads were washed and resuspended in FA lysis buffer (1mM EDTA (pH 8.0), $50 \mathrm{mM}$ HEPES-KOH (pH 7.5), $140 \mathrm{mM} \mathrm{NaCl}, 0.1 \%$ sodium deoxycholate (w/v), $1 \%$ triton X$100(\mathrm{v} / \mathrm{v}), 1 \mathrm{X}$ protease inhibitors). Two micrograms ( $\mu \mathrm{g})$ of mouse anti-dsRNA J2 (SCICONS) or mouse anti-IgG (Cell signalling) were bound to $50 \mu \mathrm{L}$ of beads for $1 \mathrm{~h}$ at room temperature. 80-90\% confluent HCT116 cells in $10 \mathrm{~cm}^{2}$ plate were washed with cold PBS. Cells were scraped, transferred to a falcon and spun at $1000 \mathrm{rpm}$ at $4^{\circ} \mathrm{C}$ for $5 \mathrm{~min}$. For each IP, cell pellet 
from one $10 \mathrm{~cm}^{2}$ plate was lysed in $100 \mu \mathrm{L}$ of Nuclei lysis buffer $(50 \mathrm{mM}$ Tris- $\mathrm{HCl}(\mathrm{pH} 8.1)$, $10 \mathrm{mM}$ EDTA (pH 8.0), $1 \%$ sodium dodecyl sulphate (SDS) (w/v), 1X protease inhibitor), incubated on ice for $10 \mathrm{~min}$ and diluted ten-fold with FA lysis buffer containing RNase inhibitor. Samples were sonicated in a BioRuptor (Diagenode) on high ("H”) for 5 min with $30 \mathrm{~s}$ on/off cycles. Following a spin at $13,000 \mathrm{rpm}$ at $4^{\circ} \mathrm{C}$ for $5 \mathrm{~min}$, supernatant was carefully transferred to a new tube and $40 \mu \mathrm{L}$ of the volume were kept as input lysate. After 1 hour of pre-clearing at $4^{\circ} \mathrm{C}$ with yeast tRNA-blocked Protein G Dynabeads, Ab conjugated beads (50 $\mu \mathrm{L}$ ) were added to the lysate and incubated for $2-3$ hours at $4^{\circ} \mathrm{C}$. Following magnetic separation, beads were washed twice with $1 \mathrm{~mL}$ of FA lysis buffer and twice with $1 \mathrm{~mL}$ of TE buffer (EDTA (10 mM, pH 8.0), Tris- $\mathrm{HCl}$ (100 mM, pH 8.0)). Beads were incubated 10 min at $95^{\circ} \mathrm{C}$ with $40 \mu \mathrm{L} 2 \mathrm{X}$ SDS Laemmli loading buffer $(120 \mathrm{mM}$ Tris/ $\mathrm{HCl} \mathrm{pH} 6.8 ; 20 \%$ glycerol; $4 \%$ SDS , $0.04 \%$ bromophenol blue; $10 \% \beta$-mercaptoethanol) and proteins were analysed either by western blot or by silver staining after electrophoretic separation on polyacrylamide SDSPAGE gel (Bio-Rad 4-20\% Criterion ${ }^{\mathrm{TM}}$ TGX Stain-Free ${ }^{\mathrm{TM}}$ Protein Gel, 18 well, \#5678094) using the SilverQuest ${ }^{\mathrm{TM}}$ Silver Staining Kit (ThermoFisher Scientific \#LC6070) according to the manufacturer's instructions.

For mass spectrometry experiments, proteins eluted from the beads were precipitated overnight with methanol containing $0.1 \%$ ammonium acetate and digested with trypsin after reduction and alkylation steps. Generated peptides were analysed by nanoLC-MS/MS on a QExactive + mass spectrometer coupled to an EASY-nanoLC-1000 (Thermo-Fisher Scientific, USA). The data were searched against a database containing the Human swissprot sequences (2017_01), the SINV and GFP sequences with a decoy strategy. Peptides were identified with Mascot algorithm (version 2.5, Matrix Science, London, UK), and the data were imported into Proline 1.4 software [http://proline.profiproteomics.fr/]. The protein identifications were validated using the following settings: Mascot pretty rank $<=1$, FDR $<=1 \%$ for PSM scores, $\mathrm{FDR}<=1 \%$ for protein set scores. The total number of MS/MS fragmentation spectra was used to quantify each protein from three independent replicates.

Mass spectrometry data obtained for each sample, including the proteins identified by the Proline software suite and their associated spectral counts $(\mathrm{SpC})$, were stored in a local MongoDB database and subsequently analysed through a Shiny Application built upon the R packages msmsEDA (Gregori J, Sanchez A, Villanueva J (2014). msmsEDA: Exploratory Data Analysis of LC-MS/MS data by spectral counts. R/Bioconductor package version 1.22.0) and msmsTests (Gregori J, Sanchez A, Villanueva J (2013). msmsTests: LC-MS/MS Differential 
Expression Tests. R/Bioconductor package version 1.22.0). Exploratory data analyses of LCMS/MS data were thus conducted and differential expression tests were performed using a negative binomial regression model. The p-values were adjusted with FDR control by the Benjamini-Hochberg method and the following criteria were used to define differentially expressed proteins: an adjusted p-value $<0.05$, a minimum of $5 \mathrm{SpC}$ in the most abundant condition, and a minimum $\log 2$ fold change of 1 .

GO term analysis was performed using the EnrichR web-based tool (http://amp.pharm.mssm.edu/Enrichr) $(49,50)$. The direct interaction network for enriched proteins was retrieved from the STRING database (https://string-db.org).

\section{SFPQ RNA Immunoprecipitation}

Four million of mock or SINV-infected HCT116 cells (MOI 0.1) were lysed 24 h post-infection using RIP immunoprecipitation buffer (50 mM Tris- $\mathrm{HCl}$ [pH 7.5], $150 \mathrm{mM} \mathrm{NaCl}, 5 \mathrm{mM}$ EDTA, $0.05 \%$ SDS, $1 \%$ triton, 1 tablet of commercial protease inhibitor cocktail (Roche)). Lysates were treated for $20 \mathrm{~min}$ at $37^{\circ} \mathrm{C}$ with $1 \mu \mathrm{L}$ of RNase-free DNase I by adjusting final concentration of $\mathrm{MgCl}_{2}(10 \mathrm{mM})$ and $\mathrm{CaCl}_{2}(5 \mathrm{mM}) .1 \mu \mathrm{L}$ of ribolock was also added.

Lysates were cleared by maximal centrifugation $10 \mathrm{~min}$ at $4^{\circ} \mathrm{C}$. Supernatants were transferred in new Eppendorf tubes. Lysates were then precleared $1 \mathrm{~h}$ at room temperature with magnetics bead treated with tRNA solution (RIP buffer $+10 \mathrm{nM}$ of tRNA).

The lysates were incubated overnight with RIP immunoprecipitation buffer containing magnetic Protein G beads conjugated with human SFPQ/PSF antibody (sc-271796, Santa Cruz, Cliniscience) or negative control mouse IgG (5415S, Cell signalling, Ozyme).

Beads were washed 3 times with RIP immunoprecipitation buffer, 3 times with wash buffer (50 mM Tris- $\mathrm{HCl}$ [pH 7.5], $200 \mathrm{mM} \mathrm{NaCl}, 5 \mathrm{mM}$ EDTA, 0.05\% SDS, $1 \%$ triton, 1 tablet of commercial protease inhibitor cocktail (Roche)) and twice with cold PBS 1X.

Immunoprecipitated RNAs and proteins were isolated by TRIzol Reagent (Invitrogen) or Laemmli loading buffer, respectively. Extracted RNAs were analysed by RT-qPCR and proteins were analysed by western blot.

\section{Western blotting}

For protein analysis in total lysates and IP, proteins were extracted by collecting cell lysates in RIPA buffer (50 mM Tris- $\mathrm{HCl}$ [pH 7.5], $150 \mathrm{mM} \mathrm{NaCl,} 5 \mathrm{mM}$ EDTA, 0.05\% SDS, $1 \%$ triton, 1 tablet of commercial protease inhibitor cocktail (Roche)). Lysates were cleared by 
centrifugation at $13000 \mathrm{rpm}$ for $30 \mathrm{~min}$ at $4^{\circ} \mathrm{C}$ to remove cell debris and the supernatant was retained for western blotting. According to the experience, $20 \mu \mathrm{g}$ of proteins of each sample or both input and eluates from the IPs were heated in Laemmli loading buffer at $95^{\circ} \mathrm{C}$ for 5 min and loaded on a SDS-polyacrylamide electrophoresis gel (Bio-Rad 4-20\% Mini-PROTEAN® TGX Stain-Free ${ }^{\mathrm{TM}}$ Protein Gels, 10 well, \#4568093). Proteins were transferred to a nitrocellulose membrane by wet transfer in 1x Tris-Glycine $+20 \%$ ethanol buffer. Membranes were blocked in 5\% milk and probed with the antibodies indicated below (Antibodies) for cellular proteins (between 1:200 and 1:5000). Viral proteins were detected using primary polyclonal antibodies against SINV CAPSID or nsP2 (kind gift from Dr Diane Griffin, Johns Hopkins University School of Medicine, Baltimore, MD). Anti-mouse-HRP (NXA931, GE Healthcare Thermo Fisher Scientific Inc.) or anti-rabbit-HRP (NA9340, GE Healthcare, Thermo Fisher Scientific Inc.) secondary antibodies were used (1:10000). Detection was performed using SuperSignal West Femto Maximum Sensitivity Substrate (Pierce, ThermoFisher Scientific) and visualized on a Fusion FX imaging system (Vilber).

\section{Immunostaining}

HCT116 cells plated on Millicell EZ 8-well slide (Merck Millipore) were fixed with 4\% formaldehyde (Sigma Aldrich), diluted in PBS 1X for $10 \mathrm{~min}$ at room temperature and incubated in blocking buffer (0.2\% Tween X-100; PBS $1 \mathrm{X} ; 5 \%$ normal goat serum) for $1 \mathrm{~h}$. Primary antibodies (see list below) were diluted in blocking buffer between 1:200 and 1:000 and incubated overnight at $4^{\circ} \mathrm{C}$. Between each step, cells were washed with PBS 1X-Tween $0.2 \%$. Cells were then incubated $1 \mathrm{~h}$ at room temperature with either goat anti-mouse Alexa 594 (A11032, Invitrogen) or goat anti-rabbit Alexa 488 (A11008, Invitrogen) secondary antibodies diluted at 1:1000 in PBS 1X-Tween 0.2\%. Cell nuclei were stained with DAPI (Life Technologies, Thermo Fisher Scientific) diluted at 1:5000 in PBS 1X during 5 min. Slides were mounted with coverslip with Fluoromount-G mounting media (Southern Biotech) and observed by confocal microscopy (LSM780, Zeiss).

\section{Antibodies}

\begin{tabular}{|l|l|l|l|}
\hline NAME & species & ref & provider \\
\hline J2 & mouse & J2 & SCICONS \\
\hline
\end{tabular}




\begin{tabular}{|c|c|c|c|}
\hline & & & $\begin{array}{l}\text { English and } \\
\text { Scientific Consulting } \\
\text { Kft, Hungary }\end{array}$ \\
\hline PKR human & rabbit & ab32506 & Abcam \\
\hline SFPQ/PSF & rabbit & ab39148 & Abcam \\
\hline SFPQ/PSF & mouse & sc-271796 & Santa Cruz \\
\hline NONO & mouse & sc-166702 & Santa Cruz \\
\hline MATRIN3 & mouse & sc-81318 & Santa Cruz \\
\hline PACT & rabbit & ab75749 & Abcam \\
\hline Histone $\mathrm{H} 3$ & rabbit & ab1791 & Abcam \\
\hline PSPC1 & mouse & sc-374181 & Santa Cruz \\
\hline ADAR & mouse & sc-271854 & Santa Cruz \\
\hline $\mathrm{IgG}$ & mouse & $5415 \mathrm{~S}$ & Cell signalling \\
\hline hNRNPA1 & mouse & sc-32301 & Santa Cruz \\
\hline Tubulin & mouse & T6557 & Sigma-Aldrich \\
\hline
\end{tabular}

\section{RNA extraction and RT-qPCR}

Total and immunoprecipitated RNAs were extracted using Tri-Reagent (Invitrogen, Fisher Scientific) according to the manufacturer's instructions. For total RNA, $1 \mu \mathrm{g}$ of RNA was reverse transcribed using SuperScript IV Vilo (Invitrogen, \#11756050) according to the manufacturer's instructions.

Real-time-PCR was performed with Maxima SYBR Green qPCR Master Mix (Applied Biosystem, \#4309155) at an annealing temperature of $60^{\circ} \mathrm{C}$ on a CFX96 touch Real-Time PCR machine (Biorad) using the following primers:

SINV genome FW: 5'-CCACTACGCAAGCAGAGACG-3';

SINV genome RV: 5'-AGTGCCCAGGGCCTGTGTCCG-3';

GAPDH FW: 5'-CTTTGGTATCGTGGAAGGACT-3';

GAPDH RV: 5' CCAGTGAGCTTCCCGTTCAG- 3'.

\section{SiRNA transfection}

Transfection complexes were prepared using ON-TARGETplus Human siRNA (Horizon), siRNAs against PKR (L-003527-00-0005), PSPC1 (L-020596-01-0005), MATR3 (L-01738200-0005), NONO (L-007756-01-0005), RBM14 (L-020144-00-0005), SFPQ (L-006455-00- 
0005), non-targeting Control Pool (D-001810-10-05) or siRNA against SINV 3'UTR (Integrated DNA Technologies) (17) at a final concentration of $50 \mathrm{nM}$ and Lipofectamine 2000 transfection reagent (Thermo Fisher Scientific, \#11668019) diluted in Opti-MEM transfection medium according to the manufacturer's instructions and they were added to 20000 HCT116 cells in 96 well plates. Two days post-transfection, cells were subjected to SINV-GFP viral infection at MOI $10^{-1}$ for 24 hours before fixation and staining with Hoechst 3342 (labelling the nucleus). GFP quantification was carried out with the INCell Analyzer 1000 Cellular Imaging and Analysis System (GE Healthcare).

Alternatively, 600000 HCT116 cells were reverse transfected with $20 \mathrm{nM}$ siRNA SFPQ or CTRL in 6-well plates, then transfected again 24 hours later and subsequently incubated for 24 hours before being infected with SINV-GFP at MOI $10^{-1}$ for 24 hours. For knock-down experiments in SK-N-BE(2) cells, 20000 cells were first transfected with $50 \mathrm{nM}$ of siRNA specific to SFPQ or CTRL in 6-well plates and 6 hours later, differentiation was induced by 10 $\mu \mathrm{M}$ retinoic acid (RA) treatment (R2625, Sigma-Aldrich). Samples were then analysed by epifluorescence microscopy, western blot, RT-qPCR or plaque assay.

\section{SFPQ CRISPR/Cas9 knock-out}

Guide RNA (gRNA) sequences targeting human SFPQ were designed using the CRISPOR tool (http://crispor.tefor.net) and cloned into pX459-V2 vector (Addgene Plasmid \#62988). Briefly, $100 \mathrm{ng}$ of digested vector and $0.25 \mu \mathrm{M}$ of the annealed oligos (guideRNA397FW: CACCGTCATCCTCCGTGATATCAGC; RV: AAACGCTGATATCACGGAGGATGAC; guideRNA64FW:

$\mathrm{RV}$ : AAACTTAGGCAGCACCCACTAACAC) were ligated and transformed into DH5 alpha cells. HCT116 cells were transfected with gRNA combined by pair. $24 \mathrm{~h}$ after, the cells were treated with $1 \mu \mathrm{g} / \mathrm{mL}$ puromycin and the surviving cells were diluted in 96 well plates to obtain 0.5 cells per well. Bulk cells were kept for validation. Two weeks later, cellular genomic DNA was extracted. Cells were lysed in 50 mM Tris-HCl pH 8.0; 100 mM EDTA pH 8.0, $100 \mathrm{mM}$ $\mathrm{NaCl}, 1 \% \mathrm{SDS}$ and $0.1 \mathrm{mg}$ of proteinase $\mathrm{K}$, and incubated overnight at $55^{\circ} \mathrm{C} .50 \mathrm{ng}$ of genomic DNA were amplified with GoTaq (Promega) using primers (IDT) able to detect the deletion, (SFPQ FW: TGGGTGTATCATCCAGTTCGG; RV: GCAGAGGTCTCTGGTGTTTGAA). PCR reactions were loaded on a $1.5 \%$ agarose gel for analysis. Wild type genomic DNA and bulk were used as controls. 


\section{EMSA analysis}

Double-stranded RNA corresponding to 65 nt-long sequence EGFP mRNA was transcribed from PCR-generated DNA templates, carrying a T7 promoter sequence and a G-residue at the transcription start site position to enhance efficiency. The following primers (IDT) were used: T7-GFP-FW: GAAATTAATACGACTCACTATAGGCAACATCCTGGGGCACAAGC;

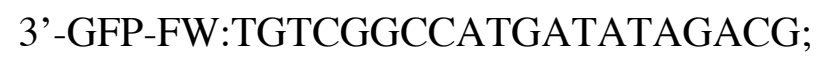

T7 RNA polymerase promoter sequence is underlined. T7-Forward and T7-Reverse templates were mixed to transcribe dsRNA. In vitro synthesis was carried out in $40 \mu \mathrm{L}$ in $40 \mathrm{mM}$ Tris$\mathrm{HCl} \mathrm{pH} \mathrm{7.5,30} \mathrm{mM} \mathrm{MgCl} 2,10 \mathrm{mM}$ DTT, $2 \mathrm{mM}$ spermidine, $0.5 \mathrm{mM}$ of each NTP, $0.1 \%$ Triton X-100, 40 U of RNase inhibitor (RiboLock, Thermo Fisher Scientific, Inc.) and $200 \mathrm{U}$ of T7 RNA polymerase (Ambion, Thermo Fisher Scientific, Inc.), for $1 \mathrm{~h} 30$ at $37^{\circ} \mathrm{C}$. Radioactive transcriptions were carried out in the same conditions except that cold UTP was at $5 \mu \mathrm{M}$ and [alpha- $\left.{ }^{32} \mathrm{P}\right]-\mathrm{UTP}$ at $425 \mu \mathrm{M}(111 \mathrm{TBq}(3000 \mathrm{Ci}) / \mathrm{mmol}$, Hartman Analytic GmbH). DNA templates were digested two times using 2 U DNase I (Thermo Fisher Scientific, Inc.) during $15 \mathrm{~min}$ at $37^{\circ} \mathrm{C}$ and free nucleotides were removed by gel filtration using $\operatorname{MicroSpin}^{\text {TN }} \mathrm{G}-25$ columns (GE-Healthcare) and RNAs were phenol extracted and ethanol-precipitated. Purified RNAs were dissolved in MilliQ water.

Binding assays were performed in $8 \mu \mathrm{L}$ in $18 \mathrm{mM}$ Tris- $\mathrm{HCl} \mathrm{pH} 7.5,75 \mathrm{mM}$ glycine, $7.5 \%$ glycerol as follows: $1000 \mathrm{cpm}$ of internally radiolabeled dsEGFP were incubated with increasing concentrations of recombinant SFPQ/PSF (OriGene Technologies) at room temperature during $30 \mathrm{~min}$. When indicated, an excess of cold RNA competitor was added prior to the SFPQ protein. RNA competitors were either the corresponding cold RNA or yeast total tRNAs. $2 \mu \mathrm{L}$ of loading dye ( $48 \%$ glycerol, $0.01 \%$ bromophenol blue) were added, and electrophoresis was carried out on native 5\% polyacrylamide gels (19:1 acrylamide/bisacrylamide ratio) in $0.5 x$ Tris-Borate-EDTA buffer at $110 \mathrm{~V}$ (cold room, $45 \mathrm{~min}$ ).

\section{Data availability}

The mass spectrometry proteomics data have been deposited to the ProteomeXchange Consortium via the PRIDE partner repository (51) with the dataset identifier PXD024554. 


\section{Acknowledgments}

The authors would like to thank all members of the Pfeffer laboratory for discussion, Amélie Weiss for technical help with GFP quantification with the INCell Analyzer 1000 Cellular Imaging System, Dr Carla Saleh for providing the SINV WT and GFP clones and Dr Diane Griffin, for providing CAPSID and nsP2 antibodies. This work was funded by the European Research Council (ERC-CoG-647455 RegulRNA) and was performed under the framework of the LABEX: ANR-10-LABX-0036_NETRNA and ANR-17-EURE-0023, which benefits from a funding from the state managed by the French National Research Agency as part of the Investments for the future program. This work has also received funding from the People Programme (Marie Curie Actions) of the European Union's Seventh Framework Program (FP7/2007-2013) under REA grant agreement $n^{\circ}$ PCOFUND-GA-2013-609102, through the PRESTIGE program coordinated by Campus France (to EG). The mass spectrometry instrumentation was funded by the University of Strasbourg, IdEx "Equipement mi-lourd" 2015 .

\section{Author contributions}

SP and EG conceived the project. SP and EG designed the work and analysed the results. EG, MM, PL, AF, JC performed the experiments. EG set up the DRIMS experiments and performed the J2-IP western blots and dot blots. JC performed the bioinformatic analysis of the proteomic data, BCWM developed the MongoDB database and the Shiny application used to analyse the mass spectrometry data, EG performed the GO-enrichment and STRING analysis. EG and MM preformed the knockdown experiments, the western blot and qPCR analysis, EG and PL performed the plaque assays. EG and MM performed the immunofluorescence and data acquisition, MM generated the SFPQ KO cell lines and set up the SFPQ-RIP, AF performed the EMSA. PL and EG performed and analysed SFPQ KD and SINV-GFP infections in differentiated SK-N-BE(2) cells. PH coordinated the proteomic experiments. EG and MM drafted the manuscript and designed the figures. EG and SP wrote the manuscript with input from the other authors. SP and EG coordinated the work. SP assured funding. All authors revised the final manuscript.

\section{References}

1. Damas ND, Fossat N, Scheel TKH. 2019. Functional Interplay between RNA Viruses and Non-Coding RNA in Mammals. Non-Coding RNA 5:7. 
2. Nagy PD, Pogany J. 2012. The dependence of viral RNA replication on co-opted host factors. Nat Rev Microbiol 10:137-149.

3. Girardi E, Pfeffer S, Baumert TF, Majzoub K. 2020. Roadblocks and fast tracks: How RNA binding proteins affect the viral RNA journey in the cell. Semin Cell Dev Biol S1084952120300914.

4. Lloyd RE. 2015. Nuclear proteins hijacked by mammalian cytoplasmic plus strand RNA viruses. Virology 479-480:457-474.

5. Novoa RR, Calderita G, Arranz R, Fontana J, Granzow H, Risco C. 2005. Virus factories: associations of cell organelles for viral replication and morphogenesis. Biol Cell 97:147172.

6. Guo Z, Li Y, Ding S-W. 2019. Small RNA-based antimicrobial immunity. Nat Rev Immunol 19:31-44.

7. Stetson DB, Medzhitov R. 2006. Type I Interferons in Host Defense. Immunity 25:373381.

8. Schlee M, Hartmann G. 2016. Discriminating self from non-self in nucleic acid sensing. Nat Rev Immunol 16:566-580.

9. Hur S. 2019. Double-Stranded RNA Sensors and Modulators in Innate Immunity. Annu Rev Immunol 37:349-375.

10. Griffin D. 2007. Alphaviruses, p 1023-1067. Fields Virol 5th Ed Lippincott Williams Wilkins Phila PA.

11. Frolova E, Gorchakov R, Garmashova N, Atasheva S, Vergara LA, Frolov I. 2006. Formation of nsP3-Specific Protein Complexes during Sindbis Virus Replication. J Virol 80:4122-4134.

12. Cristea IM, Rozjabek H, Molloy KR, Karki S, White LL, Rice CM, Rout MP, Chait BT, MacDonald MR. 2010. Host Factors Associated with the Sindbis Virus RNA-Dependent RNA Polymerase: Role for G3BP1 and G3BP2 in Virus Replication. J Virol 84:67206732.

13. Akhrymuk I, Kulemzin SV, Frolova EI. 2012. Evasion of the Innate Immune Response: the Old World Alphavirus nsP2 Protein Induces Rapid Degradation of Rpb1, a Catalytic Subunit of RNA Polymerase II. J Virol 86:7180-7191.

14. Luis Carrasco, Miguel Sanz, Esther González-Almela. 2018. The Regulation of Translation in Alphavirus-Infected Cells. Viruses 10:70.

15. Sokoloski KJ, Dickson AM, Chaskey EL, Garneau NL, Wilusz CJ, Wilusz J. 2010. Sindbis Virus Usurps the Cellular HuR Protein to Stabilize Its Transcripts and Promote Productive Infections in Mammalian and Mosquito Cells. Cell Host Microbe 8:196-207.

16. Scheel TKH, Luna JM, Liniger M, Nishiuchi E, Rozen-Gagnon K, Shlomai A, Auray G, Gerber M, Fak J, Keller I, Bruggmann R, Darnell RB, Ruggli N, Rice CM. 2016. A Broad RNA Virus Survey Reveals Both miRNA Dependence and Functional Sequestration. Cell Host Microbe 19:409-423.

17. López P, Girardi E, Mounce BC, Weiss A, Chane-Woon-Ming B, Messmer M, Kaukinen P, Kopp A, Bortolamiol-Becet D, Fendri A, Vignuzzi M, Brino L, Pfeffer S. 2020. HighThroughput Fluorescence-Based Screen Identifies the Neuronal MicroRNA miR-124 as a Positive Regulator of Alphavirus Infection. J Virol 94:e02145-19, /jvi/94/9/JVI.0214519.atom.

18. Garcia-Moreno M, Järvelin AI, Castello A. 2018. Unconventional RNA-binding proteins step into the virus-host battlefront. Wiley Interdiscip Rev RNA 9:e1498.

19. LaPointe AT, Gebhart NN, Meller ME, Hardy RW, Sokoloski KJ. 2018. Identification and Characterization of Sindbis Virus RNA-Host Protein Interactions. J Virol 92:e0217117, /jvi/92/7/e02171-17.atom. 
20. Varjak M, Saul S, Arike L, Lulla A, Peil L, Merits A. 2013. Magnetic Fractionation and Proteomic Dissection of Cellular Organelles Occupied by the Late Replication Complexes of Semliki Forest Virus. J Virol 87:10295-10312.

21. Garcia-Moreno M, Noerenberg M, Ni S, Järvelin AI, González-Almela E, Lenz CE, Bach-Pages M, Cox V, Avolio R, Davis T, Hester S, Sohier TJM, Li B, Heikel G, Michlewski G, Sanz MA, Carrasco L, Ricci EP, Pelechano V, Davis I, Fischer B, Mohammed S, Castello A. 2019. System-wide Profiling of RNA-Binding Proteins Uncovers Key Regulators of Virus Infection. Mol Cell 74:196-211.e11.

22. Bonin M, Oberstraß J, Lukacs N, Ewert K, Oesterschulze E, Kassing R, Nellen W. 2000. Determination of preferential binding sites for anti-dsRNA antibodies on double-stranded RNA by scanning force microscopy. RNA 6:563-570.

23. Schonborn J, Oberstra $\beta$ J, Breyel E, Tittgen J, Schumacher J, Lukacs N. 1991. Monoclonal antibodies to double-stranded RNA as probes of RNA structure in crude nucleic acid extracts. Nucleic Acids Res 19:2993-3000.

24. Dhir A, Dhir S, Borowski LS, Jimenez L, Teitell M, Rötig A, Crow YJ, Rice GI, Duffy D, Tamby C, Nojima T, Munnich A, Schiff M, de Almeida CR, Rehwinkel J, Dziembowski A, Szczesny RJ, Proudfoot NJ. 2018. Mitochondrial double-stranded RNA triggers antiviral signalling in humans. Nature 560:238-242.

25. White E, Schlackow M, Kamieniarz-Gdula K, Proudfoot NJ, Gullerova M. 2014. Human nuclear Dicer restricts the deleterious accumulation of endogenous double-stranded RNA. Nat Struct Mol Biol 21:552-559.

26. Nakagawa S, Yamazaki T, Hirose T. 2018. Molecular dissection of nuclear paraspeckles: towards understanding the emerging world of the RNP milieu. Open Biol 8:180150.

27. Yarosh CA, Iacona JR, Lutz CS, Lynch KW. 2015. PSF: nuclear busy-body or nuclear facilitator?: Polypyrimidine tract-binding protein-associated splicing factor. Wiley Interdiscip Rev RNA 6:351-367.

28. Gil A, Sharp PA, Jamison SF, Garcia-Blanco MA. 1991. Characterization of cDNAs encoding the polypyrimidine tract-binding protein. Genes Dev 5:1224-1236.

29. Patton JG, Mayer SA, Tempst P, Nadal-Ginard B. 1991. Characterization and molecular cloning of polypyrimidine tract-binding protein: a component of a complex necessary for pre-mRNA splicing. Genes Dev 5:1237-1251.

30. Iida K, Hagiwara M, Takeuchi A. 2020. Multilateral Bioinformatics Analyses Reveal the Function-Oriented Target Specificities and Recognition of the RNA-Binding Protein SFPQ. iScience 23:101325.

31. Zhang Z, Carmichael GG. 2001. The Fate of dsRNA in the Nucleus. Cell 106:465-476.

32. Hentze MW, Castello A, Schwarzl T, Preiss T. 2018. A brave new world of RNA-binding proteins. Nat Rev Mol Cell Biol 19:327-341.

33. Akhrymuk I, Frolov I, Frolova EI. 2016. Both RIG-I and MDA5 detect alphavirus replication in concentration-dependent mode. Virology 487:230-241.

34. Liu G, Gack MU. 2020. Distinct and Orchestrated Functions of RNA Sensors in Innate Immunity. Immunity 53:26-42.

35. You F, Sun H, Zhou X, Sun W, Liang S, Zhai Z, Jiang Z. 2009. PCBP2 mediates degradation of the adaptor MAVS via the HECT ubiquitin ligase AIP4. Nat Immunol 10:1300-1308.

36. Cao P, Luo W-W, Li C, Tong Z, Zheng Z-Q, Zhou L, Xiong Y, Li S. 2019. The heterogeneous nuclear ribonucleoprotein hnRNPM inhibits RNA virus-triggered innate immunity by antagonizing RNA sensing of RIG-I-like receptors. PLOS Pathog 15:e1007983. 
37. Wu Y, Zhao W, Liu Y, Tan X, Li X, Zou Q, Xiao Z, Xu H, Wang Y, Yang X. 2018. Function of HNRNPC in breast cancer cells by controlling the dsRNA-induced interferon response. EMBO J 37.

38. Lim YW, James D, Huang J, Lee M. 2020. The Emerging Role of the RNA-Binding Protein SFPQ in Neuronal Function and Neurodegeneration. Int J Mol Sci 21:7151.

39. Kula A, Gharu L, Marcello A. 2013. HIV-1 pre-mRNA commitment to Rev mediated export through PSF and Matrin 3. Virology 435:329-340.

40. Landeras-Bueno S, Jorba N, Pérez-Cidoncha M, Ortín J. 2011. The Splicing Factor Proline-Glutamine Rich (SFPQ/PSF) Is Involved in Influenza Virus Transcription. PLoS Pathog 7:e1002397.

41. Greco-Stewart VS, Thibault CS-L, Pelchat M. 2006. Binding of the polypyrimidine tractbinding protein-associated splicing factor (PSF) to the hepatitis delta virus RNA. Virology 356:35-44.

42. Flather D, Nguyen JHC, Semler BL, Gershon PD. 2018. Exploitation of nuclear functions by human rhinovirus, a cytoplasmic RNA virus. PLOS Pathog 14:e1007277.

43. Zhou B, Wu F, Han J, Qi F, Ni T, Qian F. 2019. Exploitation of nuclear protein SFPQ by the encephalomyocarditis virus to facilitate its replication. Biochem Biophys Res Commun 510:65-71.

44. Imamura K, Imamachi N, Akizuki G, Kumakura M, Kawaguchi A, Nagata K, Kato A, Kawaguchi Y, Sato H, Yoneda M, Kai C, Yada T, Suzuki Y, Yamada T, Ozawa T, Kaneki K, Inoue T, Kobayashi M, Kodama T, Wada Y, Sekimizu K, Akimitsu N. 2014. Long Noncoding RNA NEAT1-Dependent SFPQ Relocation from Promoter Region to Paraspeckle Mediates IL8 Expression upon Immune Stimuli. Mol Cell 53:393-406.

45. Protter DSW, Parker R. 2016. Principles and Properties of Stress Granules. Trends Cell Biol 26:668-679.

46. Kim SS-Y, Sze L, Lam K-P. 2019. The stress granule protein G3BP1 binds viral dsRNA and RIG-I to enhance interferon- $\beta$ response. J Biol Chem 294:6430-6438.

47. An H, Tan JT, Shelkovnikova TA. 2019. Stress granules regulate stress-induced paraspeckle assembly. J Cell Biol 218:4127-4140.

48. An H, Shelkovnikova TA. 2019. Stress granules regulate paraspeckles: RNP granule continuum at work. Cell Stress 3:385-387.

49. Chen EY, Tan CM, Kou Y, Duan Q, Wang Z, Meirelles G, Clark NR, Ma'ayan A. 2013. Enrichr: interactive and collaborative HTML5 gene list enrichment analysis tool. BMC Bioinformatics 14:128.

50. Kuleshov MV, Jones MR, Rouillard AD, Fernandez NF, Duan Q, Wang Z, Koplev S, Jenkins SL, Jagodnik KM, Lachmann A, McDermott MG, Monteiro CD, Gundersen GW, Ma'ayan A. 2016. Enrichr: a comprehensive gene set enrichment analysis web server 2016 update. Nucleic Acids Res 44:W90-W97.

51. Perez-Riverol Y, Csordas A, Bai J, Bernal-Llinares M, Hewapathirana S, Kundu DJ, Inuganti A, Griss J, Mayer G, Eisenacher M, Pérez E, Uszkoreit J, Pfeuffer J, Sachsenberg T, Yılmaz Ş, Tiwary S, Cox J, Audain E, Walzer M, Jarnuczak AF, Ternent T, Brazma A, Vizcaíno JA. 2019. The PRIDE database and related tools and resources in 2019: improving support for quantification data. Nucleic Acids Res 47:D442-D450. 


\section{Figures legends}

Figure 1. Identification of the dsRNA-associated proteome upon SINV infection in HCT116 cells. A) Schematic representation of SINV RNA replication. Capped and polyadenylated viral genomic RNA is represented in black, while viral antigenomic RNA is in green. Grey arrows represent dsRNA replication. ORF1 codes for the non-structural proteins necessary for RNA replication (in orange). ORF2 codes for the structural proteins (in purple). B) Confocal immunofluorescence analysis in mock and SINV infected HCT116 cells (24 hpi, MOI 0.1). Anti-dsRNA J2 antibody is in red. DAPI was used to stain the nuclei (in blue). Magnification X20(X4). C) Schematic representation of the DRIMS experimental approach. D) RT-qPCR on SINV genomic RNA upon dsRNA-IP (J2-IP) compared to CTRL-IP (IgG-IP) infected samples. Enrichment on three independent IP from three biological replicates are shown. Error bars represent mean +/- standard deviation (SD). E) Volcano plot of dsRNA-IP (J2-IP) in SINV-infected versus mock condition using data from three replicates. Red and blue dots represent proteins which are significantly enriched or depleted (adjusted p-value $<0.05$, $\operatorname{abs}(\log 2 \mathrm{FC})>1)$, respectively, in the infected samples compared to the uninfected ones. Viral non-structural and structural proteins are indicated in orange and purple, respectively. Known dsRNA binding proteins are indicated by a black square. Proteins present in paraspeckles are indicated in light blue and by a black hexagon.

Figure 2. Validation of dsRNA enriched proteins upon SINV infection in HCT116 cells.

A) GO term enrichment analysis of the dsRNA-associated proteins overrepresented in SINVJ2-IP compared to mock-J2-IP using the Enrichr software. Enriched GO terms of molecular functions (green), cellular components (yellow) and biological processes (purple) are respectively sorted by p-value. B) STRING interaction network of the significantly enriched proteins upon SINV dsRNA-IP. Nuclear and cytoplasmic RBPs are indicated in blue and red, respectively. C) Representative western blots performed on total lysate (INPUT), J2 or IgG control IP in mock and SINV-GFP-infected cells. Histone 3 (H3) and HNRNPA1 were used as negative controls. * corresponds to an unspecific band.

Figure 3. SFPQ binds to dsRNA in vitro and in vivo upon infection. A) EMSA of synthetic dsRNA with the SFPQ recombinant protein. Internally radiolabeled dsEGFP RNA $(\sim 2 \mathrm{nM})$ was incubated without protein (lanes 1 and 6) or in the presence of recombinant SFPQ (lanes 
2 to 5: 50, 100, 200 and $400 \mathrm{nM}$; lanes 7 and 8: 200 and $400 \mathrm{nM}$ ). Unlabeled dsEGFP RNA or yeast total tRNAs (ytRNA) were added as specific and unspecific competitors, respectively, at $20(+)$ and $100 \mathrm{nM}(++)$, prior to the SFPQ protein (400 nM) (lanes 9 to 12). ds = double strand. ss = single strand. B) Representative dot blot revealed using the anti-dsRNA J2 antibody on RNA extracted from total lysate (INPUT, four 10-fold dilutions), SFPQ- or IgG control-IP in mock (upper part) and SINV-infected (lower part) HCT116 cells. C) RT-qPCR on SFPQ-IP and IgG CTR-IP samples by using oligos to amplify SINV genomic RNA. Independent data from three biological replicates are shown. Error bars represent mean $+/$ - standard deviation (SD). D) Representative anti-SFPQ western blot performed on total lysate (INPUT), J2- or IgG control-IP in mock and SINV infected HCT116 cells with or without RNase T1 treatment before elution. Antibodies against PKR (positive control, dsRBP) and AGO2 (negative control, ssRBP) were used. E) Confocal immunofluorescence analysis on mock and SINV infected HCT116 cells. Antibodies against SFPQ (in green) or dsRNA (J2 antibody, in red) were used. DAPI was used to stain the nuclei (in blue). Magnification 40X. Yellow squares: 4X higher magnification.

Figure 4. SFPQ knock-down reduces SINV-GFP infection in HCT116 cells. A-D) Effect of SFPQ knockdown on SINV in HCT116 cells. A) SiRNA-based knockdown of paraspecklerelated proteins based on GFP fluorescence measurement of SINV-GFP infected HCT116 cells. SiRNA against PKR mRNA (dark grey bar) or SINV RNA (black bar) were used as positive controls. Data from three independent biological replicates are shown relative to siCTRL (negative control, white bar). B) Representative western blot performed on mock and SINV-infected cells upon siCTRL and siSFPQ treatment. Antibodies directed against SFPQ and the viral capsid protein were used. GAPDH was used as loading control. C) Fold change of SINV-GFP viral production upon siCTRL and siSFPQ transfection measured by plaque assay on three independent biological replicates. D) RT-qPCR on SINV genomic RNA relative to GAPDH upon siSFPQ treatment compared to siCTRL, in mock and SINV-GFP infected HCT116 cells. Data from three independent biological replicates are shown relative to siCTRL. Error bars in (A), (C) and (D) represent mean +/- standard deviation (SD) of three independent experiments, $* * * \mathrm{p}<0.001, * * * * \mathrm{p}<0.0001$, unpaired Student's $\mathrm{t}$ test.

Figure 5. SFPQ CRISPR/Cas9 knockout in HCT116 cells reduces SINV-GFP infection.

A) Schematic representation of the CRISPR/Cas9 knockout strategy used to generate the SFPQ 
+/- HCT116 cells. Two gRNAs spanning the exon2/intron2 junction were used to direct Cas9 cleavage and the deletion of about $300 \mathrm{bp}$ including the intron 25 'splice site (5'ss). B) Agarose gel showing the PCR fragments corresponding to the WT and KO alleles respectively. Sequencing of the PCR fragment corresponding to the KO allele is shown in (A). C-D-E) Effect of SFPQ heterozygous knockout in HCT116 cells. C) Representative pictures of SINVGFP infected cells in WT and SFPQ+/- HCT116 cells. GFP expression was measured by microscopy. BF, brightfield D) Representative western blot performed on mock and infected cells in WT and SFPQ+/- HCT116 cells. Antibodies directed against SFPQ and the viral capsid protein were used. Tubulin was used as loading control. E) Viral production of SINV-GFP in WT and SFPQ+/- HCT116 cells measured by plaque assay on three independent biological replicates. $* * * \mathrm{p}<0.001$, unpaired Student's $\mathrm{t}$ test. 


\section{Supplemental data}

\section{Supplemental table and figures legends}

Table S1 Details of the differential expression analysis results obtained for the enriched proteins in SINV-J2-IP versus mock-J2-IP.

Figure S1. DsRNA-associated protein profiles in mock and upon SINV infection in HCT116 cells A) Silver-stained 4-20\% Tris-SDS-PAGE gel of the total lysates (INPUT) and eluates from $\mathrm{J} 2$ or IgG control IP in mock and SINV-infected cells used for proteomic analysis. B) Hierarchical clustering of the J2-IP SINV (in orange) and mock (in green) infected samples. C-D) Volcano plot of (C) dsRNA-IP (J2-IP) versus IgG control-IP in SINV-infected and (D) in mock-infected conditions using data from three replicates. Red and blue dots represent proteins which are significantly enriched or depleted (adjusted p-value $<0.05$ and abs(Log2FC) $>1$ ), respectively, in the infected samples compared to the uninfected ones. Viral non-structural proteins are indicated in orange. Known dsRNA binding proteins are indicated by a black square. Proteins present in paraspeckles are indicated by a black circle.

Figure S2. Sub-cellular localization of selected dsRNA IP-enriched proteins in mock and SINV infected HCT116 cells. Confocal immunofluorescence analysis on HCT116 cells in mock and SINV infection conditions. Antibodies against selected factors were used (red or green signal in the merge). Antibody against the viral non-structural protein nsP2 was used as a positive control of infection. DAPI was used to stain the nuclei (in blue in the merge). Magnification X20(X4).

Figure S3. SFPQ binding to dsRNA. A) SFPQ binding to dsRNA in vitro. Silver-stained 4$20 \%$ Tris-SDS-PAGE gel of recombinant SFPQ (300 ng, lane 2) showing the purity of the protein (OriGene Technologies). Lane 1: protein ladder. B) SFPQ immunoprecipitation on mock and SINV-infected HCT116 samples analysed by western blot with anti-SFPQ antibody. * corresponds to IgG L and $\mathrm{H}$ chains. C) RNA analysis on agarose gel upon RNase T1 treatment on mock and SINV infected total lysate. 
Figure S4. SFPQ knockdown reduces SINV-GFP infection in SK-N-BE(2) cells. A) Representative pictures of SINV-GFP infected cells in siCTRL and siSFPQ treated cells. GFP expression was measured by microscopy. BF, brightfield. B) Representative western blot performed on non-transfected (NT) mock and infected cells and on SINV-GFP infected cells upon siCTRL and siSFPQ treatment. Antibodies directed against SFPQ and the viral capsid protein were used. Tubulin was used as loading control. C) Viral production of SINV-GFP upon siCTRL and siSFPQ transfection measured by plaque assay on three independent biological replicates. Error bars represent mean $+/-\mathrm{SD}$ of three independent experiments, ** $\mathrm{p}$ $<0.01$, unpaired Student's t test. 
A

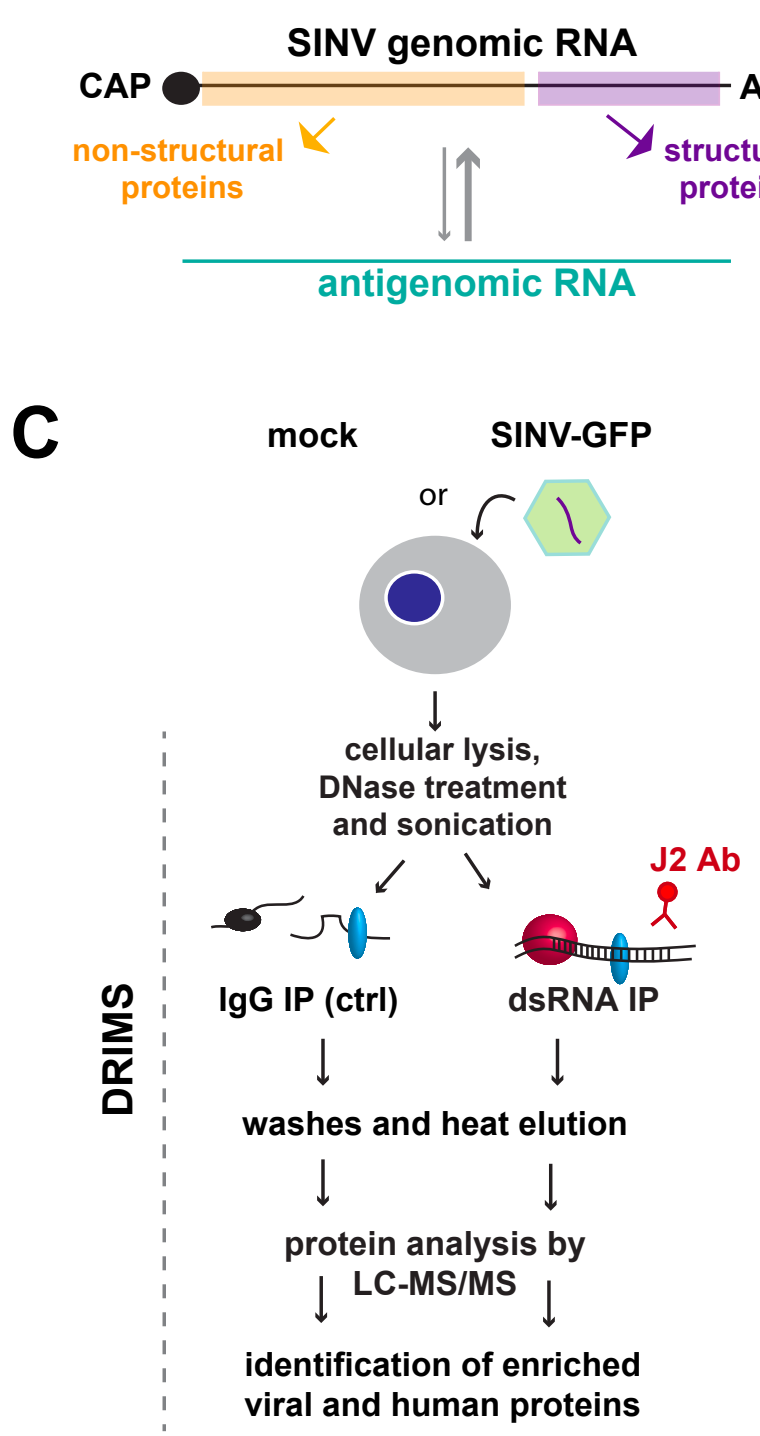

D

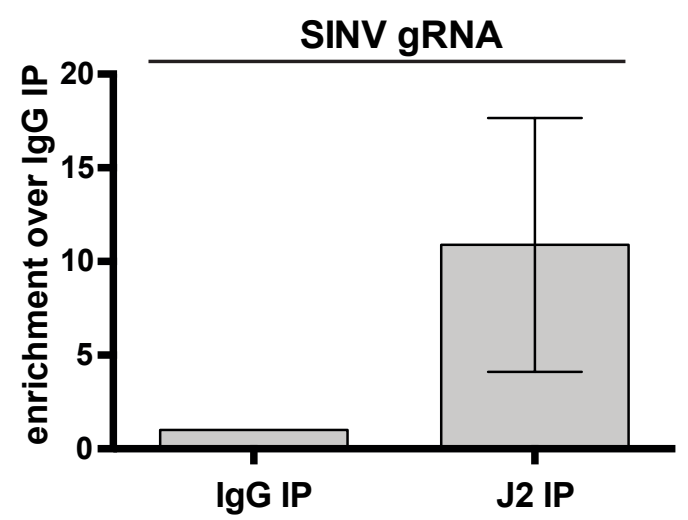

B

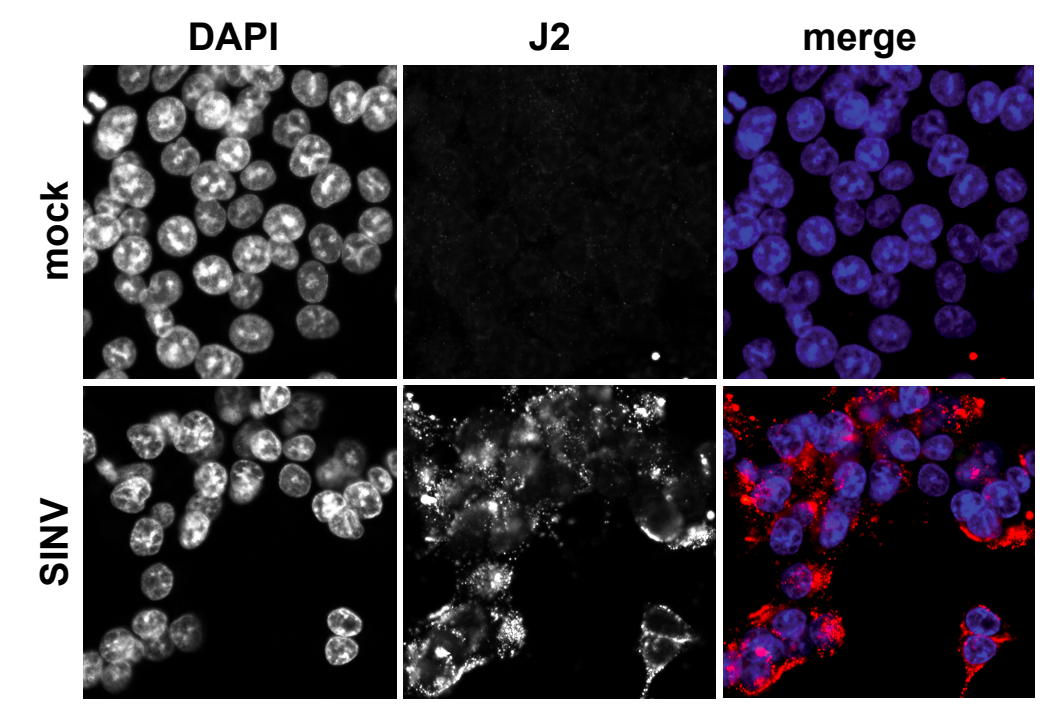

E

\section{J2 IP_SINV vs mock}

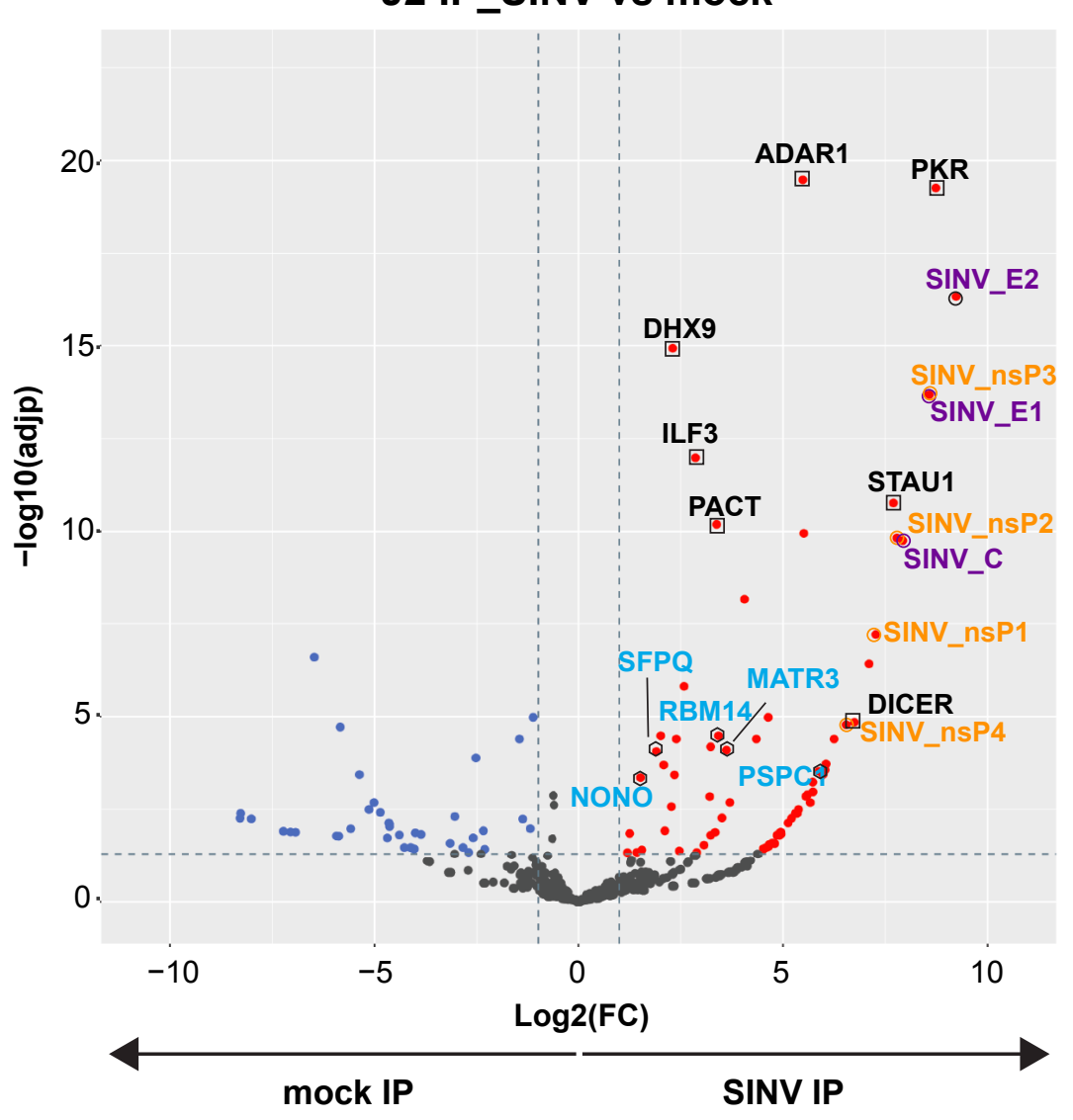




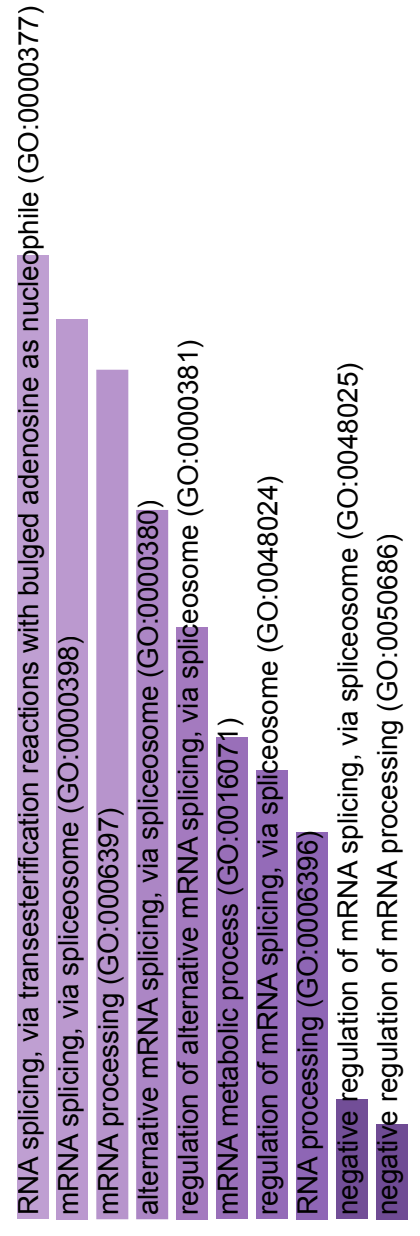

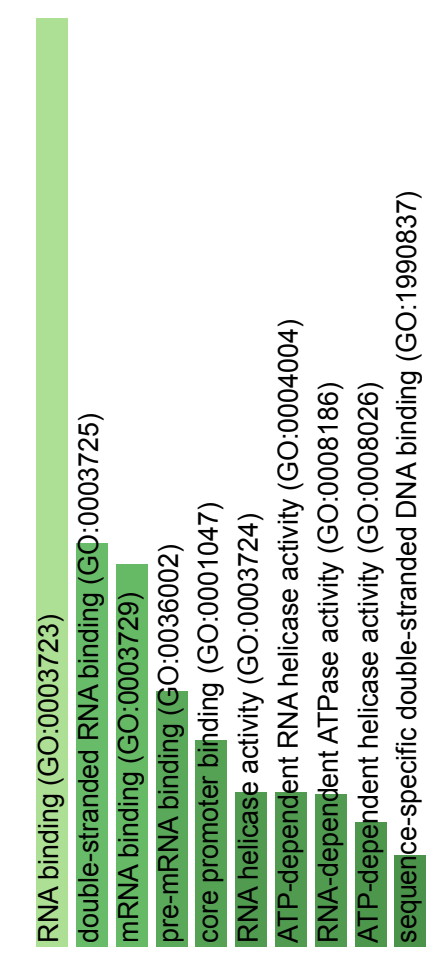

GO Molecular Function

(sorted by p-value)

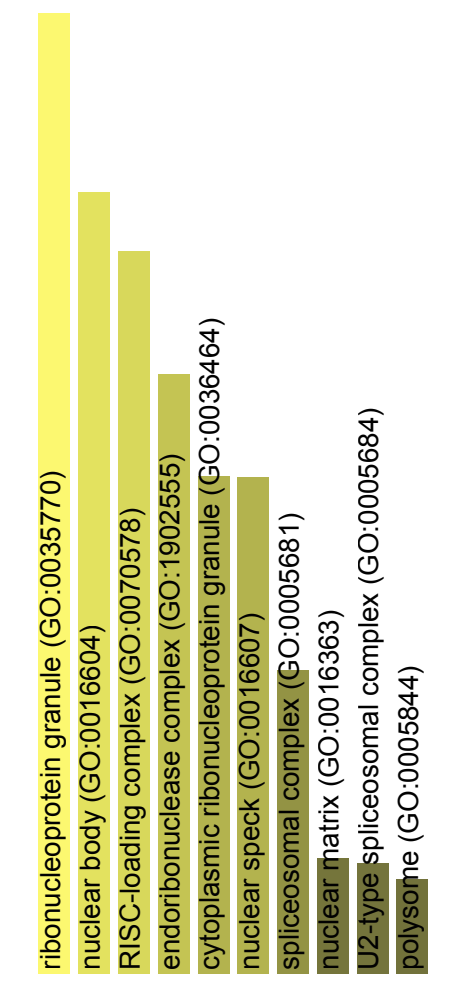

GO Cellular components

(sorted by p-value)
GO Biological Processes

(sorted by p-value)

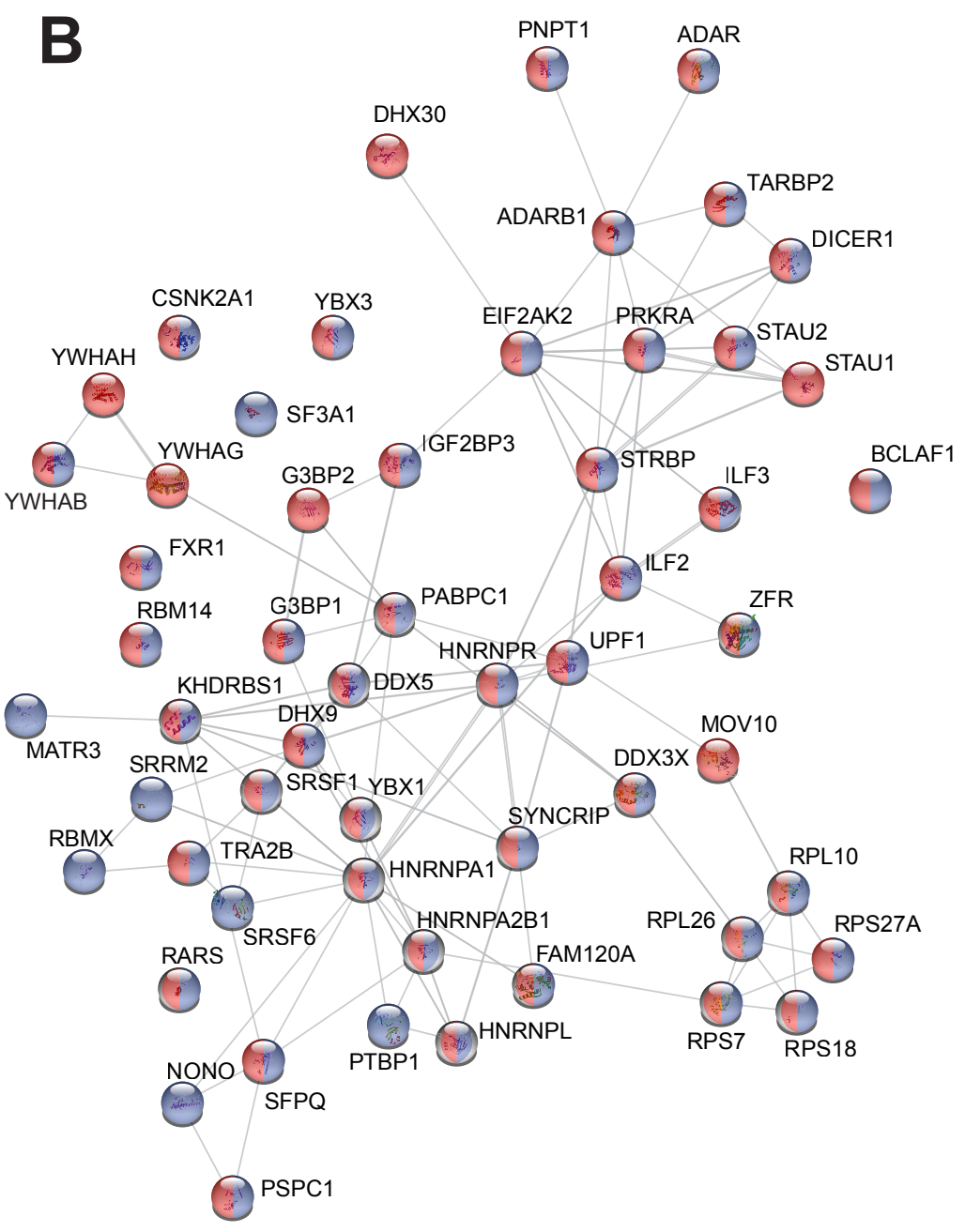

C
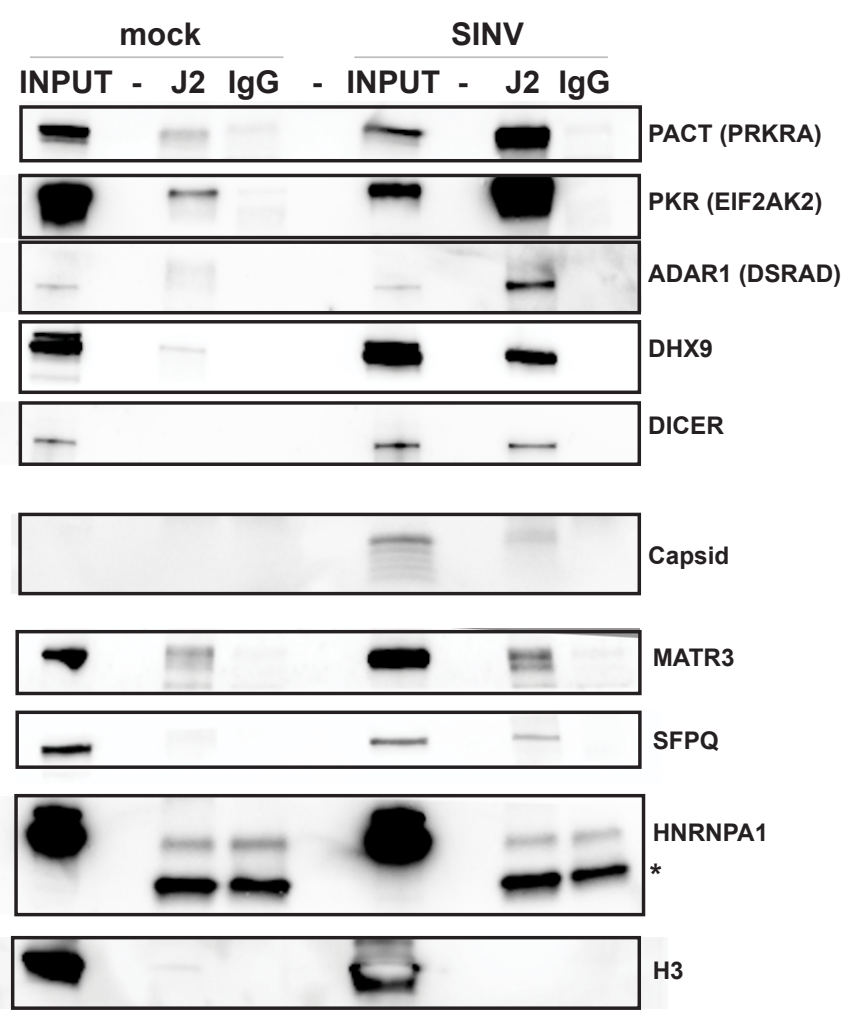
A

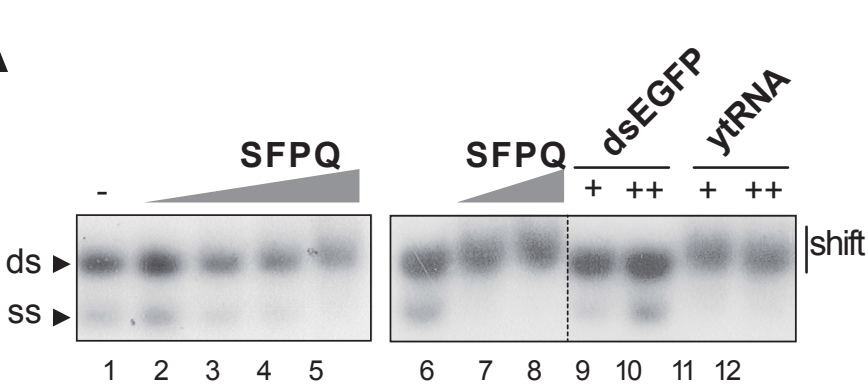

C

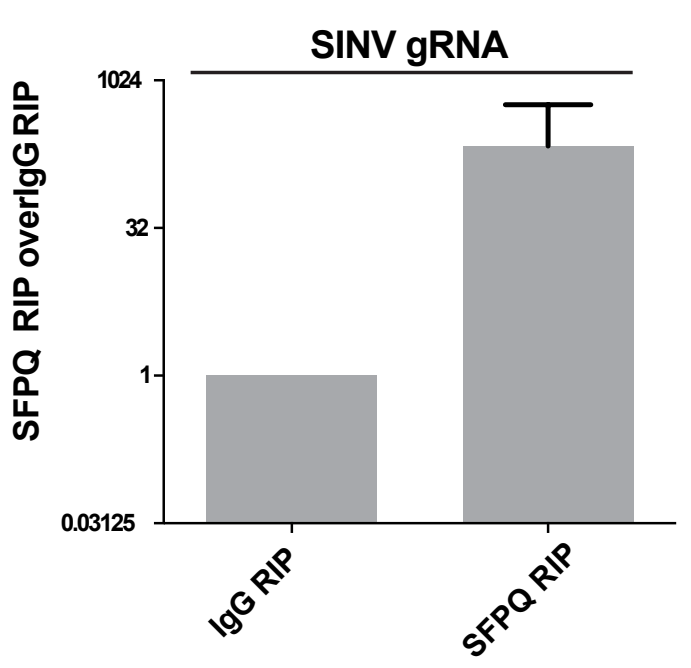

E

DAPI

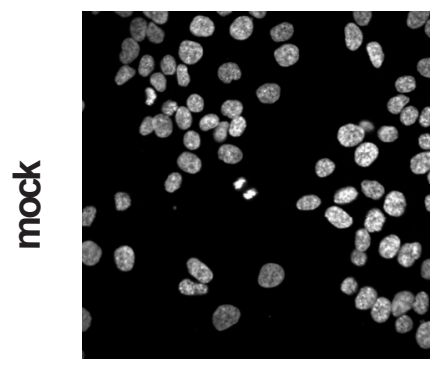

J 2
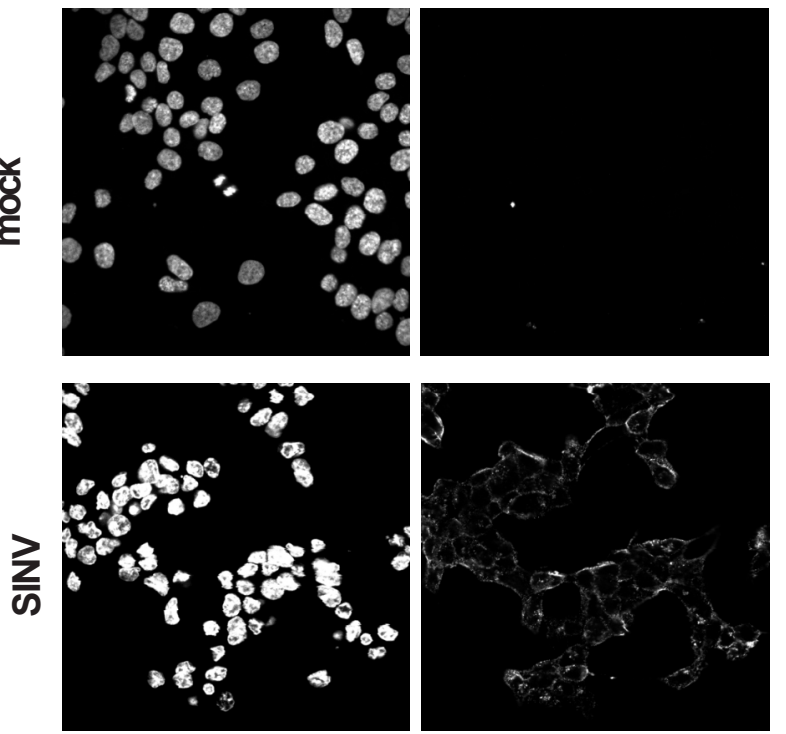

B

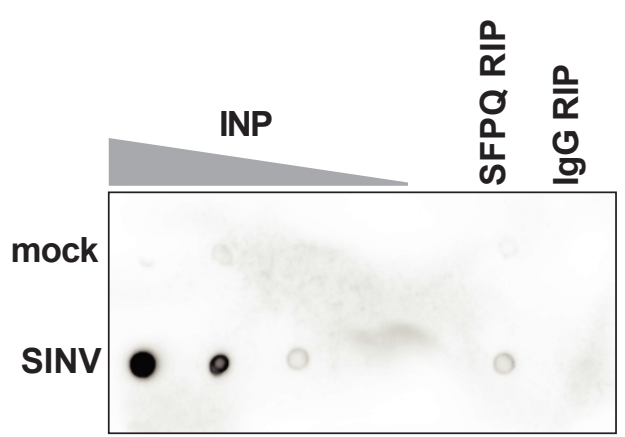

Anti-J2

D

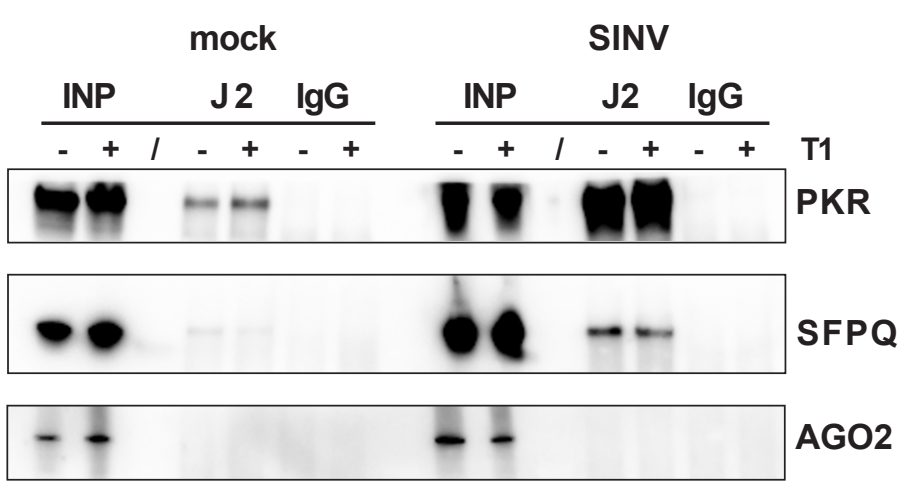

SFPQ
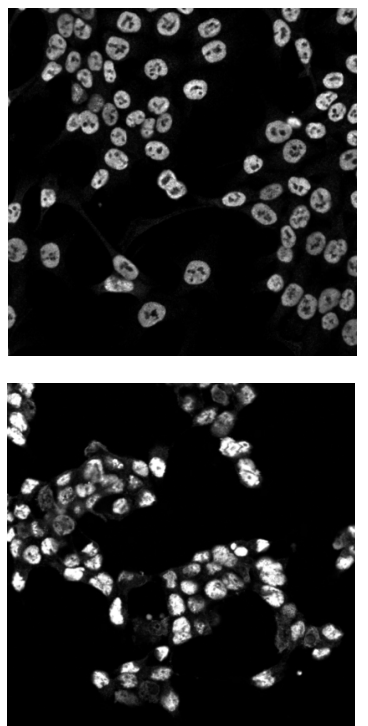

Merge
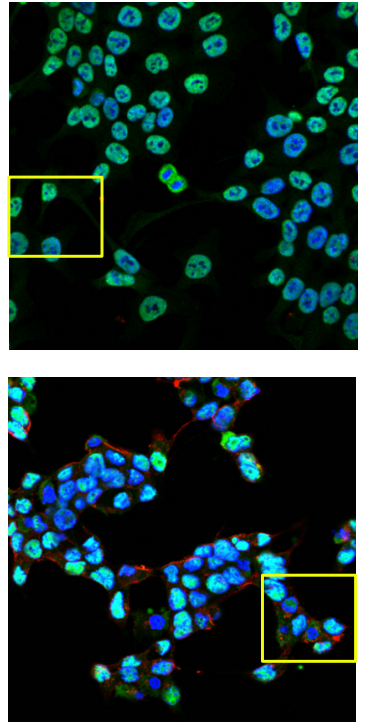

4X zoom
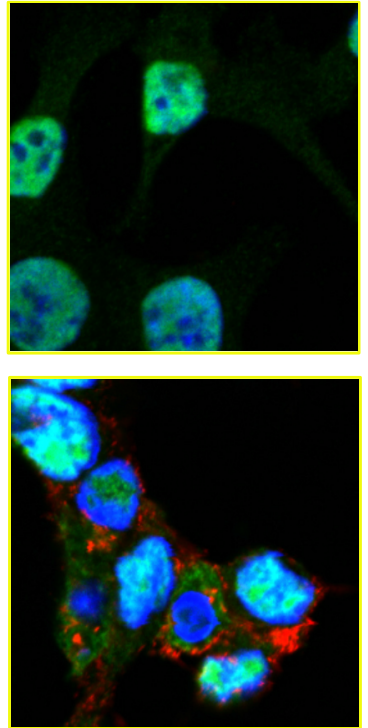
A

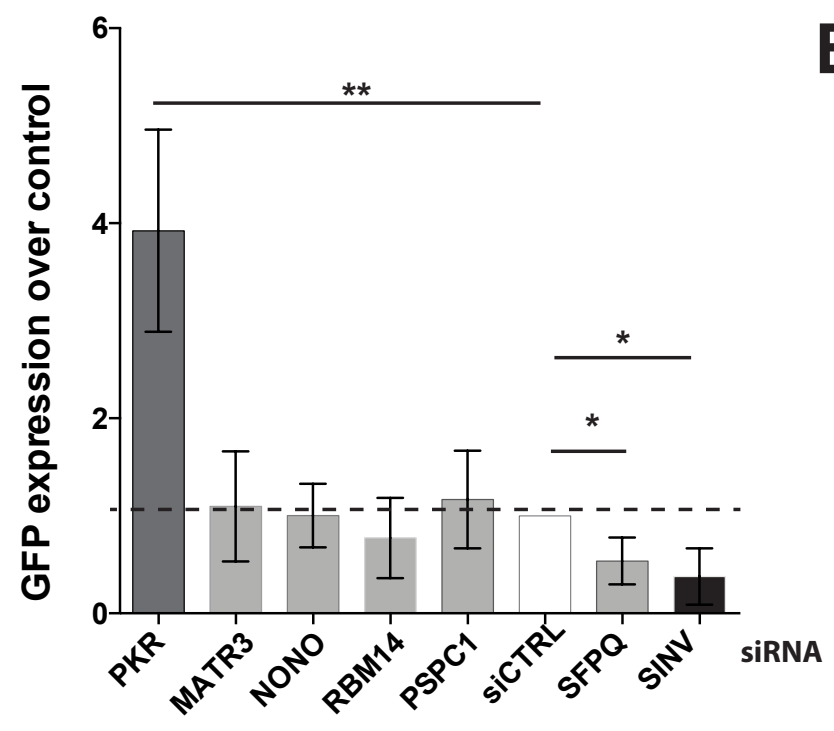

C

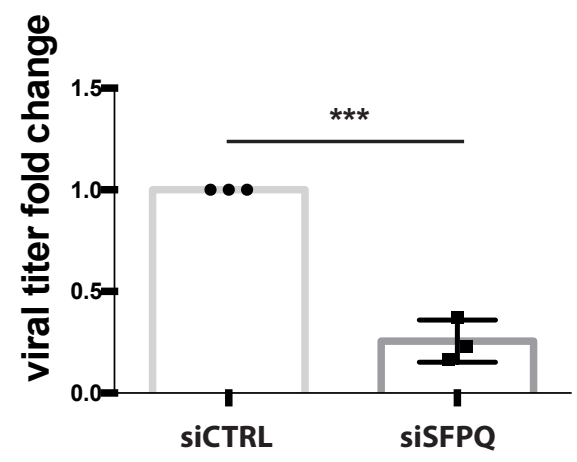

B

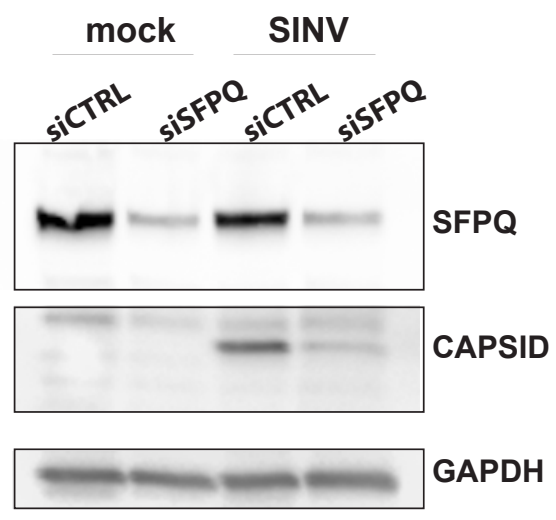

D

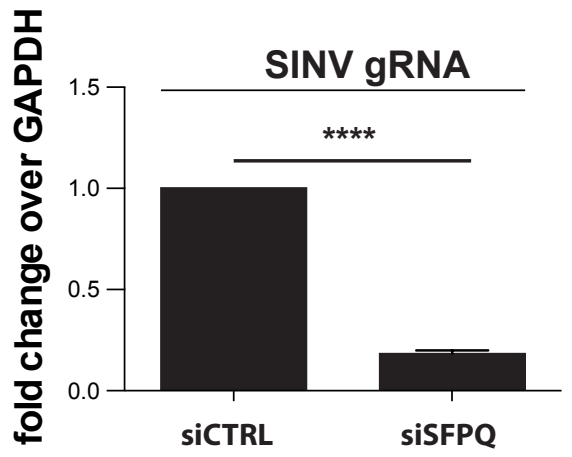


A

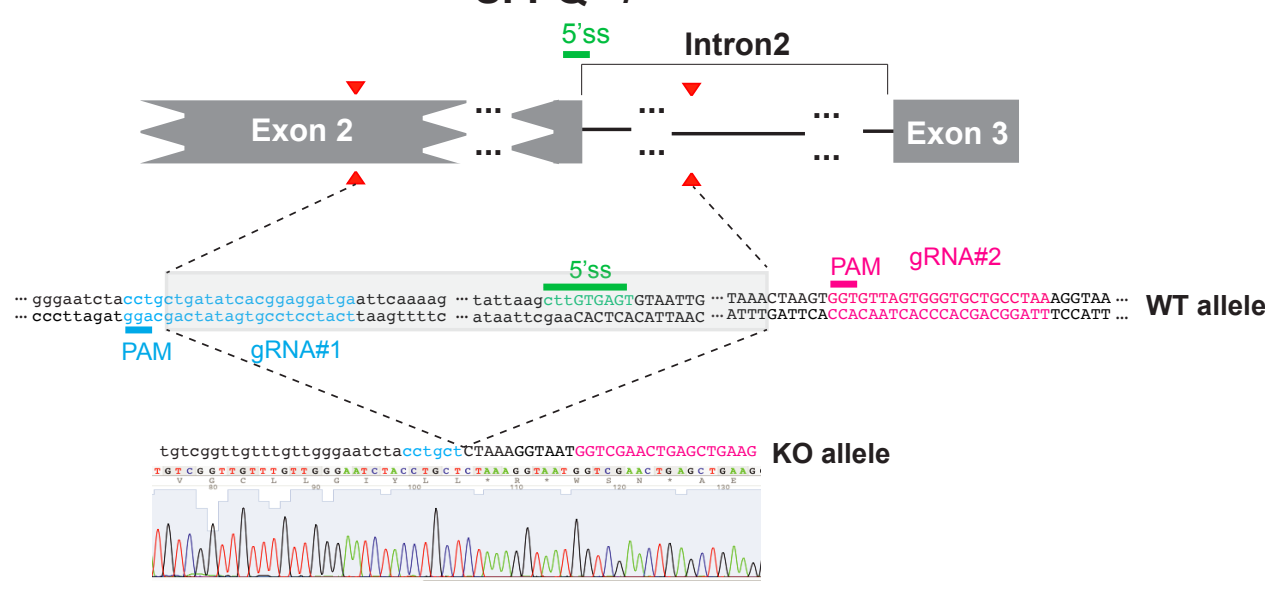

C

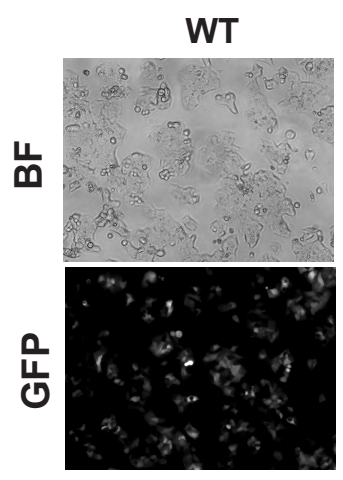

D

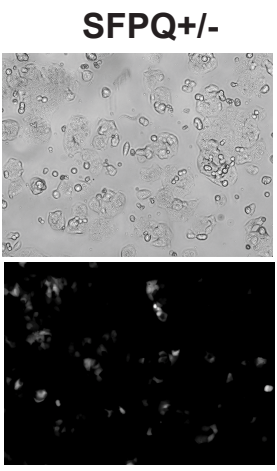

B

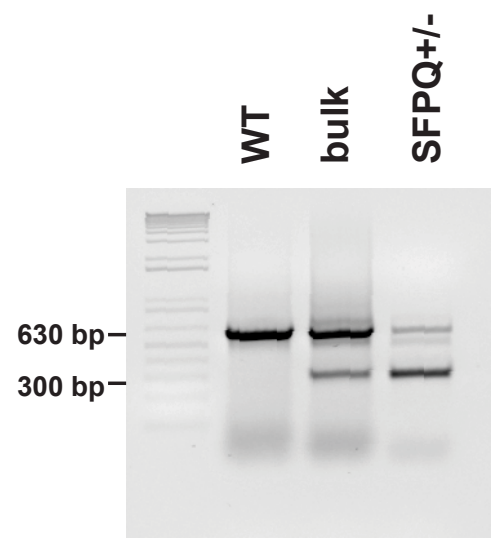

E

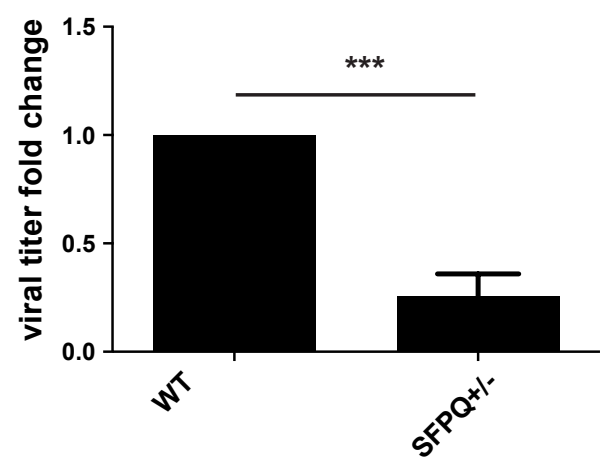

